TRANSACTIONS OF THE

AMERICAN MATHEMATICAL SOCIETY

Volume 354, Number 11, Pages 4393-4419

S 0002-9947(02)03065-9

Article electronically published on June 24, 2002

\title{
AN APPLICATION OF THE LITTLEWOOD RESTRICTION FORMULA TO THE KOSTANT-RALLIS THEOREM
}

\author{
JEB F. WILLENBRING
}

\begin{abstract}
Consider a symmetric pair $(G, K)$ of linear algebraic groups with $\mathfrak{g} \cong \mathfrak{k} \oplus \mathfrak{p}$, where $\mathfrak{k}$ and $\mathfrak{p}$ are defined as the +1 and -1 eigenspaces of the involution defining $K$. We view the ring of polynomial functions on $\mathfrak{p}$ as a representation of $K$. Moreover, set $\mathcal{P}(\mathfrak{p})=\bigoplus_{d=0}^{\infty} \mathcal{P}^{d}(\mathfrak{p})$, where $\mathcal{P}^{d}(\mathfrak{p})$ is the space of homogeneous polynomial functions on $\mathfrak{p}$ of degree $d$. This decomposition provides a graded $K$-module structure on $\mathcal{P}(\mathfrak{p})$. A decomposition of $\mathcal{P}^{d}(\mathfrak{p})$ is provided for some classical families $(G, K)$ when $d$ is within a certain stable range.

The stable range is defined so that the spaces $\mathcal{P}^{d}(\mathfrak{p})$ are within the hypothesis of the classical Littlewood restriction formula. The Littlewood restriction formula provides a branching rule from the general linear group to the standard embedding of the symplectic or orthogonal subgroup. Inside the stable range the decomposition of $\mathcal{P}^{d}(\mathfrak{p})$ is interpreted as a $q$-analog of the Kostant-Rallis theorem.
\end{abstract}

\section{INTRODUCTION}

Let $K$ be a reductive linear algebraic group over $\mathbb{C}$. Let $\left\{U^{\lambda}\right\}_{\lambda \in \hat{K}}$ be the set of distinct representatives of the irreducible regular representations of $K$. If $\mathcal{W}$ is a graded representation with the $d^{t h}$ graded component denoted $W^{d}$, the $q^{-}$ multiplicity of an irreducible regular representation $U^{\lambda}$ of $K$ in $\mathcal{W}$ is defined to be the formal power series

$$
p(\lambda, q)=\sum_{d=0}^{\infty} \operatorname{dim} \operatorname{Hom}_{K}\left(U^{\lambda}, W^{d}\right) q^{d} .
$$

Let $\operatorname{char}_{q} \mathcal{W}$ be the formal series in which the coefficient of $q^{d}$ is the character of $W^{d}$. Consequently,

$$
\operatorname{char}_{q} \mathcal{W}=\sum_{\lambda \in \hat{K}} p(\lambda, q) \chi^{\lambda},
$$

where $\chi^{\lambda}$ is the character of $U^{\lambda}$.

Our main example is as follows. Let $(\rho, V)$ be a representation of $K$. Let $\mathcal{P}(V)$ be the representation of $K$ consisting of the complex-valued polynomial functions

Received by the editors October 22, 2001.

2000 Mathematics Subject Classification. Primary 22E47, 20G05, 05 E05.

Key words and phrases. Kostant-Rallis theorem, Littlewood restriction formula, skew Schur polynomial.

This research was funded by the Yale Gibbs Instructorship as well as the NSF VIGRE postdoctoral fellowship. 
on $V$, under the usual action given by $(g \cdot f)(v)=f\left(\rho\left(g^{-1}\right)(v)\right)$ for $f \in \mathcal{P}(V), v \in V$ and $g \in K$.

Observe that we have a grading by degree, and let $\mathcal{P}^{d}(V)$ denote the degree $d$ homogeneous polynomial functions on $V$. Consider the case when $K$ is a semisimple linear algebraic group with Lie algebra $\mathfrak{k}$. $K$ acts by the adjoint representation on $\mathfrak{k}$. Let $\mathcal{I}_{\mathfrak{k}}$ be the ideal generated by the $K$-invariant polynomials on $\mathfrak{k}$ that vanish at the origin. Set $\mathcal{I}_{\mathfrak{k}}^{d}=\mathcal{P}^{d}(\mathfrak{k}) \cap \mathcal{I}_{\mathfrak{k}}$. Let $\mathcal{H}_{\mathfrak{k}}^{d}$ be the $K$-invariant complement of $\mathcal{I}_{\mathfrak{k}}^{d}$ in the representation $\mathcal{P}^{d}(\mathfrak{k})$. Set $\mathcal{H}_{\mathfrak{k}}=\bigoplus \mathcal{H}_{\mathfrak{k}}^{d}$. We will call $\mathcal{H}_{\mathfrak{k}}$ the harmonic polynomials on $\mathfrak{k}$. In [5], Kostant proved that $\mathcal{P}(\mathfrak{k})$ is a free module over the $K$ invariant polynomials, $\mathcal{P}(\mathfrak{k})^{K}$. That is to say, $\mathcal{P}(\mathfrak{k})=\mathcal{P}(\mathfrak{k})^{K} \otimes \mathcal{H}_{\mathfrak{k}}$.

Furthermore, Kostant's result also provides a formula for the multiplicity of irreducible representations of $K$ in $\mathcal{H}_{\mathfrak{k}}$. Indeed, if $T$ is a maximal torus in $K$ and $V$ is an irreducible regular representation of $K$, then the multiplicity of an irreducible representation $V$ in $\mathcal{H}_{\mathfrak{k}}$ is the dimension of the space of $T$-invariant vectors in $V$, denoted $V^{T}$.

The space $\mathcal{H}_{\mathfrak{k}}$ inherits a grading from $\mathcal{P}(\mathfrak{k})$. Nineteen years later, in [3], Hesselink provided a $q$-multiplicity formula as well. Another proof of this formula is given in [9.

A more general situation is as follows. Let $\theta$ denote a regular involution on a complex reductive linear algebraic group $G$ with differential at the identity (also denoted by) $\theta$. Let $K$ be the subgroup of $G$ consisting of the fixed points of $\theta$. Denote the Lie algebra of $K$ by $\mathfrak{k}$. We take $\mathfrak{p}$ to be the -1 eigenspace of $\theta$.

Let $\mathcal{I}_{\mathfrak{p}}$ be the ideal generated by the $K$-invariant polynomials on $\mathfrak{p}$ that vanish at the origin. Set $\mathcal{I}_{\mathfrak{p}}^{d}=\mathcal{P}^{d}(\mathfrak{p}) \cap \mathcal{I}_{\mathfrak{p}}$. Let $\mathcal{H}_{\mathfrak{p}}^{d}$ be the $K$-invariant complement of $\mathcal{I}_{\mathfrak{p}}^{d}$ in the representation $\mathcal{P}^{d}(\mathfrak{p})$. Set $\mathcal{H}_{\mathfrak{p}}=\bigoplus \mathcal{H}_{\mathfrak{p}}^{d}$. We will call $\mathcal{H}_{\mathfrak{p}}$ the harmonic polynomials on $\mathfrak{p}$. In [4], Kostant and Rallis proved the following theorem:

Theorem 1.1 (Kostant, Rallis). The space of polynomial functions on $\mathfrak{p}$ is a free module over the ring of $K$-invariant polynomials. That is,

$$
\mathcal{P}(\mathfrak{p}) \cong \mathcal{P}(\mathfrak{p})^{K} \otimes \mathcal{H}_{\mathfrak{p}},
$$

and furthermore, as a representation of $K, \mathcal{H}_{\mathfrak{p}}$ is equivalent to the representation algebraically induced from the trivial representation of a subgroup $M$ to $K$, where $M$ is the centralizer in $K$ of an Abelian subalgebra $\mathfrak{a}$ of $\mathfrak{g}$ maximal among such algebras both contained in $\mathfrak{p}$ and containing only semisimple elements.

A proof of this theorem can be found in [2].

The fact that $\mathcal{H}_{\mathfrak{p}}$ is an induced representation and Frobenius reciprocity determine the multiplicity of an irreducible representation of $K$ in the space of harmonics. Indeed, let $V^{M}$ denote the space of $M$-invariant vectors in $V$, where $V$ is an irreducible representation of $K$. Then,

$$
\operatorname{dim} \operatorname{Hom}_{K}\left(V, \mathcal{H}_{\mathfrak{p}}\right)=\operatorname{dim} V^{M}
$$

Remark 1.2. In [2], it is explained that if $G=G_{1} \times G_{1}$ and $\theta\left(g_{1}, g_{2}\right)=\left(g_{2}, g_{1}\right)$, then $K \cong G_{1}$ and $\mathfrak{k} \cong \mathfrak{p} \cong \mathfrak{g}_{1}$. In this sense, the Kostant-Rallis theorem specializes to Kostant's theorem.

The problem addressed in this paper is the $q$-multiplicity of irreducible representations of $K$ in $\mathcal{H}_{\mathfrak{p}}$ and $\mathcal{H}_{\mathfrak{k}}$ for certain pairs $(G, K)$. In [9] several examples are addressed where we do have such a graded multiplicity formula in the same sense 
of 3. Included in these examples is the case of $(G L(2 k), S p(k))$. A family of examples not completely understood is the pair $(G L(n), O(n))$ for $n \geq 5$. In order to understand these examples better, we develop a certain stable range in which the graded components of the harmonic polynomials can be completely decomposed.

The pairs addressed in this paper are $((G L(n), O(n)))$, $(G L(2 n), S p(n))$, $(O(n+k), O(n) \times O(k))$ and $(S p(n+k), S p(n) \times S p(k))$. In each of these examples we describe a stable range in $\mathcal{H}_{\mathfrak{p}}$ and $\mathcal{H}_{\mathfrak{k}}$ where a formula for the $q$-multiplicity is provided by certain symmetric functions identities.

In the case $G=O(n+k)$ and $K=O(n) \times O(k)$ the Kostant-Rallis theorem does not apply directly because $G$ is not connected. Nevertheless, $\mathcal{P}(\mathfrak{p})$ is a free module over $\mathcal{P}(\mathfrak{p})^{K}$. This case is addressed in Section 6 .

Remark 1.3. Note that the $q$-multiplicity in $\mathcal{H}_{\mathfrak{k}}$ for $(O(n+k), O(n) \times O(k))$ and $(S p(n+k), S p(n) \times S p(k))$ reduces to the $(G L(n), O(n))$ and $(G L(2 k), S p(k))$ cases.

At the end of this introduction is a table summarizing these cases.

1.1. Notation. By a partition $\lambda$ we mean a finite sequence of weakly decreasing positive integers, $\lambda_{1} \geq \lambda_{2} \geq \cdots \geq \lambda_{l}$. (In the case when there exists an $i$ such that the integer $\lambda_{i}<0$, we will refer to the sequence as an integer partition.) The number of terms in the sequence $\lambda$ will be called the length of $\lambda$ and denoted $l(\lambda)$. Partitions (or integer partitions) will always be denoted by lower case Greek letters. Let $|\lambda|=\sum_{i} \lambda_{i}$ denote the size of $\lambda$. Given a partition $\lambda$, we denote the conjugate partition to $\lambda$ by $\lambda^{\prime}$ - that is, the partition obtained by flipping the Young diagram of $\lambda$ over the main diagonal. Equivalently, $\left(\lambda^{\prime}\right)_{i}=\left\{j: \lambda_{j} \geq i\right\}$. Note that $|\lambda|=\left|\lambda^{\prime}\right|$ and $l(\lambda)=\left(\lambda^{\prime}\right)_{1}$. The following sets of partitions are fundamental for the results in this paper:

$$
\begin{aligned}
& P_{R}=\left\{\lambda: \lambda_{i} \in 2 \mathbb{Z} \text { for all } i\right\}, \\
& P_{C}=\left\{\lambda:\left(\lambda^{\prime}\right)_{i} \in 2 \mathbb{Z} \text { for all } i\right\} .
\end{aligned}
$$

The set $P_{R}$ (resp. $P_{C}$ ) consists of partitions whose Young diagrams have even rows (resp. columns). The sets $P_{R}$ and $P_{C}$ will be central to this work.

The statement of the main theorem will involve defining certain formal power series in terms of symmetric function theory. The theory of symmetric functions is highly developed (see [8] ). We recall the ingredients which we need briefly. Let $S_{n}$ denote the symmetric group on $n$ letters. The ring $\mathbb{C}\left[x_{1}, \cdots, x_{n}\right]^{S_{n}}$ of symmetric polynomials in $x_{1}, \cdots, x_{n}$ will be denoted by $\Lambda_{n}$. This ring is graded by degree; so let $\Lambda_{n}^{d}$ denote the degree $d$ homogeneous elements of $\Lambda_{n}$. For a partition $\lambda$ with $l(\lambda) \leq n$ we view $\lambda$ as an $n$-tuple by $\left(\lambda_{1}, \cdots, \lambda_{l(\lambda)}, 0, \cdots, 0\right)$. Let $\mathrm{x}$ denote the vector of variables $x_{1} x_{2} \cdots x_{n}$ and $\rho=(n-1, n-2, \cdots, 0)$. Let

$$
s_{\lambda}(\mathrm{x})=\frac{\sum_{\sigma \in S_{n}} \operatorname{sgn}(\sigma) \sigma\left(\mathrm{x}^{\lambda+\rho}\right)}{\prod_{i<j}\left(x_{i}-x_{j}\right)},
$$

where $\sigma\left(\mathrm{x}^{\lambda+\rho}\right)=x_{\sigma(1)}^{\lambda_{1}+\rho_{1}} \cdots x_{\sigma(n)}^{\lambda_{n}+\rho_{n}}$. It is a standard fact that for $\lambda$ such that $l(\lambda) \leq n$ and $|\lambda|=d, s_{\lambda}(\mathrm{x}) \in \Lambda_{n}^{d}$. Furthermore, the set of $s_{\lambda}(\mathrm{x})$ is a basis for $\Lambda_{n}^{d}$ (with $|\lambda|=d$ and $l(\lambda) \leq n$ ).

Remark 1.4. A consequence of the Weyl character formula is that $s_{\lambda}(\mathrm{x})$ is the character of the irreducible representation of $G L(n)$ evaluated on the diagonal matrix with diagonal entries $x_{1}, \cdots, x_{n}$. From this point of view it is clear that $s_{\lambda}(\mathrm{x}) \in \Lambda_{n}$, 
since the the coefficients of the monomials can be interpreted as weight multiplicities.

In $[8$, Section I.2, it is explained how we pass to a ring of symmetric functions in infinitely many variables $x_{1}, x_{2}, x_{3}, \cdots$ by setting up an inverse limit,

$$
\Lambda^{d}=\lim _{\leftarrow} \Lambda_{n}^{d} .
$$

Then, set $\Lambda:=\bigoplus_{d} \Lambda^{d}$. In this way $\Lambda$ is given a graded ring structure. From this point of view, $s_{\lambda}(\mathrm{x})$ has a precise meaning in infinitely many variables, $\mathrm{x}=$ $x_{1}, x_{2}, x_{3}, \cdots$ (see [8], p. 40, Section I.3).

Definition 1.5. Consider the following four products:

$$
\begin{aligned}
& H_{R R}(q, \mathrm{x})=\prod_{l=1}^{\infty}\left(\prod_{i \leq j} \frac{1}{1-q^{l} x_{i} x_{j}}\right), \\
& H_{R C}(q, \mathrm{x})=\prod_{l=1}^{\infty}\left(\prod_{i \leq j} \frac{1}{1-q^{2 l-1} x_{i} x_{j}} \prod_{i<j} \frac{1}{1-q^{2 l} x_{i} x_{j}}\right), \\
& H_{C R}(q, \mathrm{x})=\prod_{l=1}^{\infty}\left(\prod_{i \leq j} \frac{1}{1-q^{2 l} x_{i} x_{j}} \prod_{i<j} \frac{1}{1-q^{2 l-1} x_{i} x_{j}}\right), \\
& H_{C C}(q, \mathrm{x})=\prod_{l=1}^{\infty}\left(\prod_{i<j} \frac{1}{1-q^{l} x_{i} x_{j}}\right) .
\end{aligned}
$$

When each of the above products is expanded into a formal power series in $q$ and $x_{1}, x_{2}, \cdots$, the coefficients of $q^{d}$ for any $d$ are symmetric polynomials. Therefore, there exists a unique expansion of each product in terms of Schur polynomials. Let $\widetilde{p}_{R R}(\mu, q)$ (resp. $\left.\widetilde{p}_{R C}(\mu, q), \widetilde{p}_{C R}(\mu, q), \widetilde{p}_{C C}(\mu, q)\right)$ be the formal series in $q$ that is the coefficient of $s_{\mu}(\mathrm{x})$ in the Schur function expansion of equation (1.8) (resp. (1.9), (1.10), (1.11)). That is,

$$
\begin{array}{ll}
H_{R R}(q, \mathrm{x})=\sum_{\mu} \widetilde{p}_{R R}(\mu, q) s_{\mu}(\mathrm{x}), & H_{R C}(q, \mathrm{x})=\sum_{\mu} \widetilde{p}_{R R}(\mu, q) s_{\mu}(\mathrm{x}), \\
H_{C R}(q, \mathrm{x})=\sum_{\mu} \widetilde{p}_{R R}(\mu, q) s_{\mu}(\mathrm{x}), & H_{C C}(q, \mathrm{x})=\sum_{\mu} \widetilde{p}_{R R}(\mu, q) s_{\mu}(\mathrm{x}) .
\end{array}
$$

In the same spirit, we also define an analog of the above for two sets of variables:

$$
\begin{aligned}
& H_{G R, G R}(q, \mathrm{x}, \mathrm{y}) \\
& :=\prod_{k=1}^{\infty}\left(\prod_{i, j} \frac{1}{1-q^{2 k-1} x_{i} y_{j}} \prod_{i \leq j} \frac{1}{\left(1-q^{2 k} x_{i} x_{j}\right)\left(1-q^{2 k} y_{i} y_{j}\right)}\right)
\end{aligned}
$$

and

$$
\begin{aligned}
& H_{G C, G C}(q, \mathrm{x}, \mathrm{y}) \\
& :=\prod_{k=1}^{\infty}\left(\prod_{i, j} \frac{1}{1-q^{2 k-1} x_{i} y_{j}} \prod_{i<j} \frac{1}{\left(1-q^{2 k} x_{i} x_{j}\right)\left(1-q^{2 k} y_{i} y_{j}\right)}\right) .
\end{aligned}
$$


When each of the above is expanded in terms of Schur functions, the coefficients are formal power series in $q$ indexed by a pair of partitions. That is to say,

$$
\begin{aligned}
H_{G R, G R}(q, \mathrm{x}, \mathrm{y}) & :=\sum_{\mu, \nu} \widetilde{p}_{G R, G R}(\mu, \nu, q) s_{\mu}(\mathrm{x}) s_{\nu}(\mathrm{y}), \\
H_{G C, G C}(q, \mathrm{x}) & :=\sum_{\mu, \nu} \widetilde{p}_{G C, G C}(\mu, \nu, q) s_{\mu}(\mathrm{x}) s_{\nu}(\mathrm{y}) .
\end{aligned}
$$

The parameters $\mu$ (and $\nu$ ) in Definition [1.5 will in general index an irreducible representation of the group $K$. Here we set up some notation for indexing the irreducible representations of $G L(n), O(n)$ and $S p(k)$.

$G L(n)$ representations. Let $\lambda$ be an integer partition with at most $n$ parts. By $F_{(n)}^{\lambda}$ we will mean the irreducible finite-dimensional representation of $G L(n)$ with highest weight given by $\lambda$. The weights are parameterized as usual by the action of the diagonal matrices in $G L(n)$.

$O(n)$ representations. The complex (or real) orthogonal group has two connected components. Because the group is disconnected, we cannot index irreducible representation by highest weights. There is, however, an analog of Schur-Weyl duality for the case of $O(n)$ in which each irreducible regular representation is indexed uniquely by a non-negative integer partition $\nu$ such that $\left(\nu^{\prime}\right)_{1}+\left(\nu^{\prime}\right)_{2} \leq n$. That is, the sum of the first two columns of the Young diagram of $\nu$ is at most $n$. (See [2], Chapter 10, for details.) Let $E_{(n)}^{\nu}$ denote the irreducible representation of $O(n)$ indexed by $\nu$ in this way.

The irreducible regular representations of $S O(n)$ may be indexed by their highest weight, since the group is a connected reductive linear algebraic group. In [2] Section 5.2.2 the irreducible representations of $O(n)$ are determined in terms of their restrictions to $S O(n)$ (which is a normal subgroup having index 2). See [2] Section 10.2.4 and 10.2.5 for the correspondence between this parameterization and the above parameterization by partitions.

$S p(k)$ representations. Let $V_{(k)}^{\nu}$ denote the irreducible regular representation of $S p(k)$ with highest weight indexed by the partition $\nu$ in the usual way.

Before we proceed it is necessary to explain some convenient notation in the context of formal power series.

Definition $1.6(\mathcal{O}$-Notation). Let $k[[q]]$ denote the ring of formal power series in an indeterminate $q$ over a field $k$. Let there be given $F_{i}(q) \in k[[q]](i=1,2)$ such that

$$
F_{i}(q)=f_{i}^{(0)}+f_{i}^{(1)} q+f_{i}^{(2)} q^{2}+\cdots+f_{i}^{(d)} q^{d}+\cdots .
$$

If $f_{1}^{(r)}=f_{2}^{(r)}$ for all $r$ with $0 \leq r \leq d$, then we will say that $F_{1}(q)=F_{2}(q)$ up to order $d$, and write

$$
F_{1}(q)=F_{2}(q)+\mathcal{O}\left(q^{d+1}\right)
$$


1.2. The Stable Range. We now describe the main result of the paper.

Theorem 1.7. The q-multiplicity of $E_{(n)}^{\nu}$ in the space $\mathcal{H}_{\mathfrak{p}}$ for the symmetric pair $(G L(n), O(n))$ is equal to $\widetilde{p}_{R R}(\nu, q)$ up to order $r$, where

$$
r=\left\lfloor\frac{n}{2}\right\rfloor .
$$

The q-multiplicity of $V_{(k)}^{\nu}$ in the space $\mathcal{H}_{\mathfrak{k}}$ for the $(G L(2 k)$, Sp $(k))$ case of Kostant's theorem is equal to $\widetilde{p}_{R C}(\nu, q)$ up to order $r$, where

$$
r=k .
$$

The q-multiplicity of $E_{(n)}^{\nu}$ in the space $\mathcal{H}_{\mathfrak{k}}$ for the $(G L(n), O(n))$ case of Kostant's theorem is equal to $\widetilde{p}_{C R}(\nu, q)$ up to order $r$, where

$$
r=\left\lfloor\frac{n}{4}\right\rfloor .
$$

The q-multiplicity of $V_{(k)}^{\nu}$ in the space $\mathcal{H}_{\mathfrak{p}}$ for the symmetric pair $(G L(2 k), S p(k))$ is equal to $\widetilde{p}_{C C}(\nu, q)$ up to order $r$, where

$$
r=\left\lfloor\frac{k}{2}\right\rfloor .
$$

The q-multiplicity of $E_{(k)}^{\mu} \otimes E_{(n)}^{\nu}$ (resp. $\left.V_{(k)}^{\mu} \otimes V_{(n)}^{\nu}\right)$ in the space $\mathcal{H}_{\mathfrak{p}}$ for the symmetric pair $(O(k+n), O(k) \times O(n))$ (resp. $(S p(k+n), S p(k) \times S p(n)))$ is equal to $\widetilde{p}_{G R, G R}(\mu, \nu, q)$ (resp. $\left.\widetilde{p}_{G C, G C}(\mu, \nu, q)\right)$ up to order $r$, where

$$
r= \begin{cases}\left\lfloor\frac{1}{2} \min (k, n)\right\rfloor & \text { for } O(k) \times O(n), \\ \min (k, n) & \text { for } S p(k) \times S p(n) .\end{cases}
$$

Remark 1.8. Part (1.18) of Theorem 1.7 appeared in the author's thesis (see 10]), which was written under the direction of Professor Nolan Wallach.

Remark 1.9. We summarize the cases addressed in Theorem 1.7 in the following table.

\begin{tabular}{|c|c|c|c|}
\hline$q$-Series & Representation & $K$ & Stability range $\leq r$ in $\mathcal{H}, r=$ \\
\hline$p_{R R}$ & $S^{2} \mathbb{C}^{n}$ & $O(n)$ & $\lfloor n / 2\rfloor$ \\
$p_{R C}$ & $S^{2} \mathbb{C}^{2 k}$ & $S p(k)$ & $k$ \\
$p_{C R}$ & $\wedge^{2} \mathbb{C}^{n}$ & $O(n)$ & $\lfloor n / 4\rfloor$ \\
$p_{C C}$ & $\wedge^{2} \mathbb{C}^{2 k}$ & $S p(k)$ & $\lfloor k / 2\rfloor$ \\
$p_{G R, G R}$ & $\mathbb{C}^{k} \otimes \mathbb{C}^{n}$ & $O(k) \times O(n)$ & $\lfloor\min (k, n) / 2\rfloor$ \\
$p_{G C, G C}$ & $\mathbb{C}^{2 k} \otimes \mathbb{C}^{2 n}$ & $S p(k) \times S p(n)$ & $\min (k, n)$ \\
\hline
\end{tabular}

The goal of the remainder of the paper is to provide a proof of Theorem $1.7 \mathrm{on}$ a case-by-case basis. In Section 2 we develop the Hilbert series of several rings of invariants which arise in classical invariant theory. In all cases the result is deduced from the Cartan-Helgason theorem applied to certain multiplicity-free spaces.

In Section 3 the Littlewood restriction formulas are introduced. These formulas are fundamental to the results of the paper. Briefly, these are branching multiplicity formulas for the standard inclusions $O(n) \subseteq G L(n)$ and $S p(k) \subseteq G L(2 k)$.

In Section 4 some identities in the theory of symmetric functions are developed. These identities are then related to the Kostant-Rallis theorem via the Littlewood restriction formulas in the remaining sections. Section 5 addresses the cases labelled 
RR, RC, CR, and CC, while Section [6] addresses the remaining two cases: GR,GR and GC,GC.

Acknowledgements. The author thanks Professor Nolan Wallach for insightful conversations regarding several aspects of this work.

\section{The Cartan-Helgason theorem and some consequences}

Given a graded representation $\mathcal{W}$, the Hilbert series of $\mathcal{W}$ will be a formal series in $q$ in which the coefficient of $q^{d}$ is $\operatorname{dim} W^{d}$. By $\mathcal{W}^{K}$ we denote the $K$-invariants in $\mathcal{W}$. $\mathcal{W}^{K}$ inherits a grading from $\mathcal{W}$. In this section we recall the Hilbert series for $\mathcal{W}^{K}$ in some well-known examples where the invariants are a polynomial ring.

Theorem 2.1. The Hilbert series for $\mathcal{P}(V)^{K}$ when $K=O(n)$ or $S p(k)$ and $V$ is the symmetric or exterior square of the standard representation is

$$
\begin{aligned}
& \prod_{l=1}^{n} \frac{1}{1-q^{l}} \quad \text { if } V=S^{2}\left(\mathbb{C}^{n}\right) \text { and } K=O(n), \\
& \prod_{l=1}^{k} \frac{1}{1-q^{2 l}} \quad \text { if } V=S^{2}\left(\mathbb{C}^{2 k}\right) \text { and } K=S p(k), \\
& \prod_{l=1}^{\left\lfloor\frac{n}{2}\right.} \frac{1}{1-q^{2 l}} \quad \text { if } V=\wedge^{2}\left(\mathbb{C}^{n}\right) \text { and } K=O(n), \\
& \prod_{l=1}^{k} \frac{1}{1-q^{l}} \quad \text { if } V=\wedge^{2}\left(\mathbb{C}^{2 k}\right) \text { and } K=S p(k) .
\end{aligned}
$$

Remark 2.2. It is important to note that each case of Theorem [2.1] is an instance of the Chevalley restriction theorem for $O(n)$ or $S p(k)$ (see [9]).

We will describe here some representations of $G L(n)$ which will be of interest later on in the paper, and then we will provide a proof of Theorem 2.1

As a representation of $G L(k) \times G L(n)$, the space of $k \times n$ matrices (under the action by $\left.(g, h) \cdot X=g X h^{T}\right)$ is equivalent to $\mathbb{C}^{k} \otimes \mathbb{C}^{n}$.

Proposition 2.3. Under the action $((g, h) \cdot f)(v \otimes w)=f(v g \otimes w g)$ for $(g, h) \in$ $G L(k) \times G L(n), v \otimes w \in \mathbb{C}^{k} \otimes \mathbb{C}^{n}$,

$$
\mathcal{P}^{d}\left(\mathbb{C}^{k} \otimes \mathbb{C}^{n}\right) \cong \bigoplus_{\substack{l(\lambda) \leq \min (k, n) \\|\lambda|=d}} F_{(k)}^{\lambda} \otimes F_{(n)}^{\lambda} .
$$

Proof. See 2], p. 255, Section 5.2.4.

As a representation of $G L(n)$, the space of symmetric $n \times n$ matrices (under the action $g \cdot X=g X g^{T}$ ) is equivalent to the symmetric square of the standard representation, denoted $S^{2}\left(\mathbb{C}^{n}\right) . \mathcal{P}\left(S M_{n}\right)$ is a representation of $G L(n)$ under the action given by $g \cdot f(X)=f\left(g^{T} X g\right)$ for $X \in S M_{n}, f \in \mathcal{P}\left(S M_{n}\right)$ and $g \in G L(n)$.

\section{Proposition 2.4.}

$$
\mathcal{P}^{d}\left(S^{2} \mathbb{C}^{n}\right) \cong \bigoplus_{\substack{\lambda \in P_{R}: l(\lambda) \leq n \\|\lambda|=2 d}} F_{(n)}^{\lambda} .
$$

Proof. See [2], p. 257, Section 5.2.5. 
As a representation of $G L(n)$, the space of skew-symmetric $n \times n$ matrices (under the action $g \cdot X=g X g^{T}$ ) is equivalent to the exterior square of the standard representation, denoted $\wedge^{2}\left(\mathbb{C}^{n}\right)$. $\mathcal{P}\left(A M_{n}\right)$ is a representation of $G L(n)$ under the action given by $g \cdot f(X)=f\left(g^{T} X g\right)$ for $X \in A M_{n}, f \in \mathcal{P}\left(A M_{n}\right)$ and $g \in G L(n)$.

\section{Proposition 2.5.}

$$
\mathcal{P}^{d}\left(\wedge^{2} \mathbb{C}^{n}\right) \cong \bigoplus_{\substack{\lambda \in P_{C}: l(\lambda) \leq n \\|\lambda|=2 d}} F_{(n)}^{\lambda} .
$$

Proof. See [2], p. 258, Section 5.2.6.

Theorem 2.6 (Cartan-Helgason). Given a partition $\lambda$ with at most $n$ parts,

$$
\operatorname{dim}\left(F_{(n)}^{\lambda}\right)^{O(n)}= \begin{cases}1 & \text { if } \lambda \in P_{R}, \\ 0 & \text { otherwise },\end{cases}
$$

and in the case where $n=2 k$,

$$
\operatorname{dim}\left(F_{(2 k)}^{\lambda}\right)^{S p(k)}= \begin{cases}1 & \text { if } \lambda \in P_{C} \\ 0 & \text { otherwise }\end{cases}
$$

Proof. See [2], p. 549, Section 12.3.3.

Proof of Theorem 2.1. Applying Theorem 2.6 to the decompositions in Propositions 2.4 and 2.5, we obtain

$$
\begin{aligned}
& \sum_{\substack{l(\lambda) \leq n \\
\lambda \in P_{R}}} q^{\frac{|\lambda|}{2}} \quad \text { if } V=S^{2}\left(\mathbb{C}^{n}\right) \text { and } K=O(n), \\
& \sum_{\substack{l(\lambda) \leq 2 k \\
\lambda \in P_{R}}} q^{\frac{|\lambda|}{2}} \quad \text { if } V=S^{2}\left(\mathbb{C}^{2 k}\right) \text { and } K=S p(k), \\
& \sum_{\substack{l(\lambda) \leq n \\
\lambda \in P_{C}}} q^{\frac{|\lambda|}{2}} \quad \text { if } V=\wedge^{2}\left(\mathbb{C}^{n}\right) \text { and } K=O(n), \\
& \sum_{\substack{l(\lambda) \leq 2 k \\
\lambda \in P_{C}}} q^{\frac{|\lambda|}{2}} \quad \text { if } V=\wedge^{2}\left(\mathbb{C}^{2 k}\right) \text { and } K=S p(k) .
\end{aligned}
$$

Closing the sums (2.10)-(2.13) is straightforward.

\section{The Littlewood Restriction Formula}

Definition 3.1 (Littlewood-Richardson coefficients). Given $\lambda, \mu$ and $\nu$ with $l(\lambda)$, $l(\mu), l(\nu) \leq n$,

$$
c_{\mu \nu}^{\lambda}=\operatorname{dim} \operatorname{Hom}_{G L(n)}\left(F_{(n)}^{\lambda}, F_{(n)}^{\mu} \otimes F_{(n)}^{\nu}\right) .
$$

Remark 3.2. If $\lambda, \mu$, and $\nu$ are such that $l(\lambda), l(\mu), l(\nu) \leq n_{0}$, then, for all $n \geq n_{0}$,

$$
\operatorname{dim} \operatorname{Hom}_{G L(n)}\left(F_{(n)}^{\lambda}, F_{(n)}^{\mu} \otimes F_{(n)}^{\nu}\right)=\operatorname{dim} \operatorname{Hom}_{G L\left(n_{0}\right)}\left(F_{\left(n_{0}\right)}^{\lambda}, F_{\left(n_{0}\right)}^{\mu} \otimes F_{\left(n_{0}\right)}^{\nu}\right) .
$$

In this sense 3.1 is independent of $n$. This point of view is illuminated from SchurWeyl duality (see [2]) and the theory of symmetric functions (see [8]). 
Remark 3.3. One can show from the Weyl character formula that if $c_{\mu \nu}^{\lambda} \neq 0$, then $\mu_{i}, \nu_{i} \leq \lambda_{i}$ for all $i$. In other words, the Young diagrams of $\mu$ and $\nu$ fit inside the Young diagram of $\lambda$. In the same light, if $c_{\mu \nu}^{\lambda} \neq 0$, then $|\lambda|=|\mu|+|\nu|$.

Definition 3.4. Regard $F_{(n)}^{\lambda}$ as a representation of $O(n)$ by restriction. Then let $C_{\nu}^{\lambda}$ denote the multiplicity of $E_{(n)}^{\nu}$ in $F_{(n)}^{\lambda}$. That is to say,

$$
C_{\nu}^{\lambda}=\operatorname{dim} \operatorname{Hom}_{O(n)}\left(E_{(n)}^{\nu}, F_{(n)}^{\lambda}\right) .
$$

Theorem 3.5 (Littlewood Restriction for $O(n) \subseteq G L(n)$, see [6, 7]). Given $\lambda$ such that $l(\lambda) \leq \frac{n}{2}$ and $\nu$ such that $\left(\nu^{\prime}\right)_{1}+\left(\nu^{\prime}\right)_{2} \leq n$, then

$$
C_{\nu}^{\lambda}=\sum_{\mu \in P_{R}} c_{\mu \nu}^{\lambda} .
$$

Definition 3.6. Regard $F_{(2 k)}^{\lambda}$ as a representation of $S p(k)$ by restriction. Then let $D_{\nu}^{\lambda}$ denote the multiplicity of $V_{(k)}^{\nu}$ in $F_{(2 k)}^{\lambda}$. That is to say,

$$
D_{\nu}^{\lambda}=\operatorname{dim} \operatorname{Hom}_{S p(k)}\left(V_{(k)}^{\nu}, F_{(2 k)}^{\lambda}\right) .
$$

Theorem 3.7 (Littlewood Restriction for $S p(k) \subseteq G L(2 k)$, see [6, 7]). Given $\lambda$ such that $l(\lambda) \leq k$ and $\nu$ such that $l(\nu) \leq k$, then

$$
D_{\nu}^{\lambda}=\sum_{\mu \in P_{C}} c_{\mu \nu}^{\lambda} .
$$

Notice that the hypotheses of Theorems 3.5 and 3.7 do not include an arbitrary parameter for the representation of the general linear group. These hypotheses are necessary, but for certain $\nu$ it is possible to weaken them considerably. For more discussion on this point, see [10] and [1].

\section{RESUlts FROM SYMMETRIC FUNCTION THEORY}

Proposition 4.1 (Cauchy-Littlewood).

$$
\prod_{i, j} \frac{1}{1-x_{i} y_{j}}=\sum_{\lambda} s_{\lambda}(\mathrm{x}) s_{\lambda}(\mathrm{y}) .
$$

Proof. See 8], p. 63, I.4, Equation (4.3).

Proposition 4.2.

$$
\prod_{i \leq j} \frac{1}{1-x_{i} x_{j}}=\sum_{\lambda \in P_{R}} s_{\lambda}(\mathrm{x}) .
$$

Proof. See [8], p. 77, I.5, Example 5(a).

\section{Proposition 4.3.}

$$
\prod_{i<j} \frac{1}{1-x_{i} x_{j}}=\sum_{\lambda \in P_{C}} s_{\lambda}(\mathrm{x}) .
$$

Proof. See 8], p. 77, I.5, Example 5(b).

Remark 4.4. By passing to characters, the decompositions in Proposition 2.3, 2.4 and 2.5 provide proofs for Propositions 4.14 .2 and 4.3 in the case of finitely many variables. The result for an infinite set of variables can be deduced from the finite case. 


\subsection{Skew Schur Polynomials.}

Definition 4.5. Given partitions $\lambda$ and $\mu$, define

$$
s_{\lambda / \mu}(\mathrm{x})=\sum_{\nu} c_{\mu \nu}^{\lambda} s_{\nu}(\mathrm{x}),
$$

where the $c_{\mu \nu}^{\lambda}$ are as in Definition 3.1

Let $(*, *)$ denote the Hall scalar product (defined so that the Schur polynomials are an orthonormal set (see [8])). In terms of $(*, *)$, another way to state Definition 4.5 is

$$
\left(s_{\lambda}, s_{\mu} s_{\nu}\right)=\left(s_{\lambda / \mu}, s_{\nu}\right) .
$$

In this sense the operator that takes $s_{\lambda}$ to $s_{\lambda / \mu}$ is adjoint to the operator given by multiplication by $s_{\mu}$.

By $s_{\lambda}(\mathrm{x}, \mathrm{y})$ we will mean the Schur function in the variables consisting of the union of the variable sets $\left\{x_{1}, x_{2}, \cdots\right\}$ and $\left\{y_{1}, y_{2}, \cdots\right\}$.

\section{Proposition 4.6.}

$$
s_{\lambda}(\mathrm{x}, \mathrm{y})=\sum_{\mu} s_{\lambda / \mu}(\mathrm{x}) s_{\mu}(\mathrm{y})
$$

Proof. See 8], p. 72, Chapter I, Section 5, Equation (5.9).

\subsection{Some identities.}

Proposition 4.7 ([8, p. 93, I.5, Example 27(a), Equation (4)). Let $\mu$ be a partition. Then

$$
\sum_{\lambda \in P_{R}} s_{\lambda / \mu}(\mathrm{x})=\frac{\sum_{\nu \in P_{R}} s_{\mu / \nu}(\mathrm{x})}{\prod_{i \leq j}\left(1-x_{i} x_{j}\right)} .
$$

Proof. Set

$$
R(\mathrm{x}, \mathrm{y})=\prod_{i \leq j} \frac{1}{1-x_{i} x_{j}} \prod_{i, j} \frac{1}{1-x_{i} y_{j}} \prod_{i \leq j} \frac{1}{1-y_{i} y_{j}} .
$$

We expand the above in two ways. First, viewing the concatenation of $\mathrm{x}$ with $\mathrm{y}$ as one set of variables, by Proposition 4.2 we have

$$
R(\mathrm{x}, \mathrm{y})=\sum_{\lambda \in P_{R}} s_{\lambda}(\mathrm{x}, \mathrm{y}) .
$$

By Proposition 4.6 this expression becomes

$$
\sum_{\lambda \in P_{R}}\left(\sum_{\delta} s_{\lambda / \delta}(\mathrm{x}) s_{\delta}(\mathrm{y})\right) .
$$

Reorganizing the order of summation, we obtain

$$
R(\mathrm{x}, \mathrm{y})=\sum_{\delta} s_{\delta}(\mathrm{y})\left(\sum_{\lambda \in P_{R}} s_{\lambda / \delta}(\mathrm{x})\right) .
$$


We now expand Equation (4.8) using Propositions 4.1 and 4.7 as follows:

$$
\prod_{i \leq j} \frac{1}{1-x_{i} x_{j}}\left(\sum_{\beta} s_{\beta}(\mathrm{x}) s_{\beta}(\mathrm{y})\right)\left(\sum_{\nu \in P_{R}} s_{\nu}(\mathrm{y})\right) .
$$

By Definition 3.1 the last two factors in the above expression become

$$
\left(\sum_{\beta} s_{\beta}(\mathrm{x}) s_{\beta}(\mathrm{y})\right)\left(\sum_{\nu \in P_{R}} s_{\nu}(\mathrm{y})\right)=\sum_{\substack{\nu \in P_{R} \\ \beta, \delta}} c_{\beta \nu}^{\delta} s_{\delta}(\mathrm{y}) s_{\beta}(\mathrm{x}) .
$$

By Definition 4.5 we can rewrite the above and obtain

$$
R(\mathrm{x}, \mathrm{y})=\prod_{i \leq j} \frac{1}{1-x_{i} x_{j}} \sum_{\delta} s_{\delta}(\mathrm{y})\left(\sum_{\nu} s_{\delta / \nu}(\mathrm{x})\right) .
$$

The result follows from taking the coefficients of $s_{\mu}(\mathrm{y})$ in (4.11) and (4.14).

Proposition 4.8 ([8], p. 93, I.5, Example 27(a), Equation (5)). Let $\mu$ be a partition. Then

$$
\sum_{\lambda \in P_{C}} s_{\lambda / \mu}(\mathrm{x})=\frac{\sum_{\nu \in P_{C}} s_{\mu / \nu}(\mathrm{x})}{\prod_{i<j}\left(1-x_{i} x_{j}\right)} .
$$

Proof. The proof is essentially the same as Proposition 4.7 except we use Proposition 4.8 instead of Proposition 4.7.

\section{Definition 4.9.}

$$
\begin{aligned}
A_{R R}(q, \mathrm{x}) & =\sum_{\lambda, \mu \in P_{R}} s_{\lambda / \mu}(\mathrm{x}) q^{\frac{|\lambda|}{2}}, & A_{R C}(q, \mathrm{x}) & =\sum_{\substack{\lambda \in P_{R} \\
\mu \in P_{C}}} s_{\lambda / \mu}(\mathrm{x}) q^{\frac{|\lambda|}{2}} \\
A_{C R}(q, \mathrm{x}) & =\sum_{\substack{\lambda \in P_{C} \\
\mu \in P_{R}}} s_{\lambda / \mu}(\mathrm{x}) q^{\frac{|\lambda|}{2}}, & A_{C C}(q, \mathrm{x}) & =\sum_{\lambda, \mu \in P_{C}} s_{\lambda / \mu}(\mathrm{x}) q^{\frac{|\lambda|}{2}} .
\end{aligned}
$$

The goal of this section is to establish

\section{Proposition 4.10.}

$$
\begin{aligned}
& A_{R R}(q, \mathrm{x})=\prod_{l=1}^{\infty} \frac{1}{1-q^{l}}\left(\prod_{i \leq j} \frac{1}{1-q^{l} x_{i} x_{j}}\right) \\
& A_{R C}(q, \mathrm{x})=\prod_{l=1}^{\infty} \frac{1}{1-q^{2 l}}\left(\prod_{i<j} \frac{1}{1-q^{2 l} x_{i} x_{j}} \prod_{i \leq j} \frac{1}{1-q^{2 l-1} x_{i} x_{j}}\right) \\
& A_{C R}(q, \mathrm{x})=\prod_{l=1}^{\infty} \frac{1}{1-q^{2 l}}\left(\prod_{i<j} \frac{1}{1-q^{2 l-1} x_{i} x_{j}} \prod_{i \leq j} \frac{1}{1-q^{2 l} x_{i} x_{j}}\right) \\
& A_{C C}(q, \mathrm{x})=\prod_{l=1}^{\infty} \frac{1}{1-q^{l}}\left(\prod_{i<j} \frac{1}{1-q^{l} x_{i} x_{j}}\right)
\end{aligned}
$$

The proof of Proposition 4.10 will need a few lemmas. 


\section{Lemma 4.11.}

$$
\begin{aligned}
& A_{R R}(q, \mathrm{x})=\frac{A_{R R}\left(q, q^{\frac{1}{2}} \mathrm{x}\right)}{\prod_{i \leq j}\left(1-q x_{i} x_{j}\right)}, \\
& A_{R C}(q, \mathrm{x})=\frac{A_{C R}\left(q, q^{\frac{1}{2}} \mathrm{x}\right)}{\prod_{i \leq j}\left(1-q x_{i} x_{j}\right)}, \\
& A_{C R}(q, \mathrm{x})=\frac{A_{R C}\left(q, q^{\frac{1}{2}} \mathrm{x}\right)}{\prod_{i<j}\left(1-q x_{i} x_{j}\right)}, \\
& A_{C C}(q, \mathrm{x})=\frac{A_{C C}\left(q, q^{\frac{1}{2}} \mathrm{x}\right)}{\prod_{i<j}\left(1-q x_{i} x_{j}\right)} .
\end{aligned}
$$

Proof. In each of equations (4.20) through (4.23), the proof is an application of Proposition 4.7 or 4.8 .

Equation (4.20): By definition,

$$
A_{R R}(q, \mathrm{x})=\sum_{\lambda, \mu \in P_{R}} s_{\lambda / \mu}(\mathrm{x}) q^{\frac{|\lambda|}{2}} .
$$

$s_{\lambda / \mu}(\mathrm{x})$ is a homogeneous polynomial of degree $|\lambda|-|\mu|$, and so $A_{R R}(q, \mathrm{x})$ is

$$
\sum_{\lambda, \mu \in P_{R}} s_{\lambda / \mu}\left(q^{\frac{1}{2}} \mathrm{x}\right) q^{\frac{|\mu|}{2}}
$$

Next, we rearrange the order of summation:

$$
\sum_{\mu \in P_{R}} q^{\frac{|\mu|}{2}}\left(\sum_{\lambda \in P_{R}} s_{\lambda / \mu}\left(q^{\frac{1}{2}} \mathrm{x}\right)\right) .
$$

Here we apply Proposition 4.7;

$$
\sum_{\mu \in P_{R}} q^{\frac{|\mu|}{2}}\left(\frac{\sum_{\nu \in P_{R}} s_{\mu / \nu}\left(q^{\frac{1}{2}} \mathrm{x}\right)}{\prod_{i \leq j}\left(1-\left(q^{\frac{1}{2}} x_{i}\right)\left(q^{\frac{1}{2}} x_{j}\right)\right)}\right)
$$

Reorganizing the sum, we obtain the result:

$$
\frac{\sum_{\mu \in P_{R}} \sum_{\nu \in P_{R}} s_{\mu / \nu}\left(q^{\frac{1}{2}} \mathrm{x}\right) q^{\frac{|\mu|}{2}}}{\prod_{i \leq j}\left(1-q x_{i} x_{j}\right)}=\frac{A_{R R}\left(q, q^{\frac{1}{2}} \mathrm{x}\right)}{\prod_{i \leq j}\left(1-q x_{i} x_{j}\right)} .
$$

Equation (4.21): By definition,

$$
A_{R C}(q, \mathrm{x})=\sum_{\substack{\lambda \in P_{R} \\ \mu \in P_{C}}} s_{\lambda / \mu}(\mathrm{x}) q^{\frac{|\lambda|}{2}}
$$

By homogeneity,

$$
\sum_{\substack{\lambda \in P_{R} \\ \mu \in P_{C}}} s_{\lambda / \mu}\left(q^{\frac{1}{2}} \mathrm{X}\right) q^{\frac{|\mu|}{2}}
$$

Next, we rearrange the order of summation:

$$
\sum_{\mu \in P_{C}} q^{\frac{|\mu|}{2}}\left(\sum_{\lambda \in P_{R}} s_{\lambda / \mu}\left(q^{\frac{1}{2}} \mathrm{x}\right)\right) .
$$


Here we apply Proposition 4.7:

$$
\sum_{\mu \in P_{C}} q^{\frac{|\mu|}{2}}\left(\frac{\sum_{\nu \in P_{R}} s_{\mu / \nu}\left(q^{\frac{1}{2}} \mathrm{x}\right)}{\prod_{i \leq j}\left(1-\left(q^{\frac{1}{2}} x_{i}\right)\left(q^{\frac{1}{2}} x_{j}\right)\right)}\right) .
$$

Reorganizing the sum, we obtain the result:

$$
\frac{\sum_{\mu \in P_{C}} \sum_{\nu \in P_{R}} s_{\mu / \nu}\left(q^{\frac{1}{2}} \mathrm{x}\right) q^{\frac{|\mu|}{2}}}{\prod_{i \leq j}\left(1-q x_{i} x_{j}\right)}=\frac{A_{C R}\left(q, q^{\frac{1}{2}} \mathrm{x}\right)}{\prod_{i \leq j}\left(1-q x_{i} x_{j}\right)}
$$

Equation (4.22): By definition,

$$
A_{C R}(q, \mathrm{x})=\sum_{\substack{\lambda \in P_{C} \\ \mu \in P_{R}}} s_{\lambda / \mu}(\mathrm{x}) q^{\frac{|\lambda|}{2}}=\sum_{\mu \in P_{R}} q^{\frac{|\mu|}{2}}\left(\sum_{\lambda \in P_{C}} s_{\lambda / \mu}\left(q^{\frac{1}{2}} \mathrm{x}\right)\right) .
$$

Here we apply Proposition 4.8 .

$$
\sum_{\mu \in P_{R}} q^{\frac{|\mu|}{2}}\left(\frac{\sum_{\nu \in P_{C}} s_{\mu / \nu}\left(q^{\frac{1}{2}} \mathrm{x}\right)}{\prod_{i<j}\left(1-\left(q^{\frac{1}{2}} x_{i}\right)\left(q^{\frac{1}{2}} x_{j}\right)\right)}\right)
$$

Reorganizing the sum, we obtain the result.

Equation (4.23): As in the case of Equation (4.20),

$$
A_{C C}(q, \mathrm{x})=\sum_{\lambda, \mu \in P_{C}} s_{\lambda / \mu}(\mathrm{x}) q^{\frac{|\lambda|}{2}}=\sum_{\mu \in P_{C}} q^{\frac{|\mu|}{2}}\left(\sum_{\lambda \in P_{R}} s_{\lambda / \mu}\left(q^{\frac{1}{2}} \mathrm{x}\right)\right) .
$$

Here we apply Proposition 4.8 and reorganize:

$$
\sum_{\mu \in P_{C}} q^{\frac{|\mu|}{2}}\left(\frac{\sum_{\nu \in P_{R}} s_{\mu / \nu}\left(q^{\frac{1}{2}} \mathrm{x}\right)}{\prod_{i<j}\left(1-\left(q^{\frac{1}{2}} x_{i}\right)\left(q^{\frac{1}{2}} x_{j}\right)\right)}\right)=\frac{A_{C C}\left(q, q^{\frac{1}{2}} \mathrm{x}\right)}{\prod_{i<j}\left(1-q x_{i} x_{j}\right)}
$$

Lemma 4.12.

$$
\begin{aligned}
& A_{R R}\left(q, q^{\frac{d}{2}} \mathrm{x}\right)=\prod_{l \geq 1}\left(\frac{1}{1-q^{l}}\right)+\mathcal{O}\left(q^{\left\lfloor\frac{d}{2}\right\rfloor+1}\right), \\
& A_{R C}\left(q, q^{\frac{d}{2}} \mathrm{x}\right)=\prod_{l \geq 1}\left(\frac{1}{1-q^{2 l}}\right)+\mathcal{O}\left(q^{\left\lfloor\frac{d}{2}\right\rfloor+1}\right), \\
& A_{C R}\left(q, q^{\frac{d}{2}} \mathrm{x}\right)=\prod_{l \geq 1}\left(\frac{1}{1-q^{2 l}}\right)+\mathcal{O}\left(q^{\left\lfloor\frac{d}{2}\right\rfloor+1}\right), \\
& A_{C C}\left(q, q^{\frac{d}{2}} \mathrm{x}\right)=\prod_{l \geq 1}\left(\frac{1}{1-q^{l}}\right)+\mathcal{O}\left(q^{\left\lfloor\frac{d}{2}\right\rfloor+1}\right) .
\end{aligned}
$$

Remark 4.13. If $\tilde{d} \geq d$, then clearly $F(q)=G(q)+O(\tilde{d}) \Rightarrow F(q)=G(q)+O\left(q^{d}\right)$. We point it out because we will be tacitly using this fact.

Remark 4.14. In our context the coefficient field $k$ in Definition[1.6] is to be rational functions in the countably infinite set of variables $x_{1}, x_{2}, x_{3}, \cdots$. 
Proof. In each case we expand the expression given in Definition 4.9,

Equation (4.38): The polynomials $s_{\lambda / \mu}(\mathrm{x})$ are homogeneous polynomials of degree $|\lambda|-|\mu|$. Hence,

$$
A_{R R}\left(q, q^{\frac{d}{2}} \mathrm{x}\right)=\sum_{\lambda, \mu \in P_{R}} s_{\lambda / \mu}\left(q^{\frac{d}{2}} \mathrm{x}\right) q^{\frac{|\lambda|}{2}}=\sum_{\lambda, \mu \in P_{R}} s_{\lambda / \mu}(\mathrm{x}) q^{\frac{d(|\lambda|-|\mu|)+|\lambda|}{2}} .
$$

Separate the sum into two cases: $|\lambda|=|\mu|$ and $|\lambda| \neq|\mu|$. Then,

$$
\begin{array}{r}
\sum_{\substack{\lambda, \mu \in P_{R} \\
\lambda=\mu}} s_{\lambda / \mu}(\mathrm{x}) q^{\frac{d(|\lambda|-|\mu|)+|\lambda|}{2}}+\sum_{\substack{\lambda, \mu \in P_{R} \\
\lambda \neq \mu}} s_{\lambda / \mu}(\mathrm{x}) q^{\frac{d(|\lambda|-|\mu|)+|\lambda|}{2}} \\
=\sum_{\substack{\lambda, \mu \in P_{R} \\
\lambda=\mu}} q^{\frac{|\lambda|}{2}}+\sum_{\substack{\lambda, \mu \in P_{R} \\
\lambda \neq \mu}} s_{\lambda / \mu}(\mathrm{x}) q^{\frac{d(|\lambda|-|\mu|)+|\lambda|}{2}} .
\end{array}
$$

The formal series in the first sum is easily seen to be

$$
\prod_{l=1}^{\infty} \frac{1}{1-q^{l}}
$$

The lowest power of $q$ occurring in the second term is obtained when $|\lambda|-|\mu|=1$. In this case, the power is not less than $\left\lfloor\frac{d}{2}\right\rfloor+1$. Hence, Equation (4.43) is

$$
\prod_{l=1}^{\infty} \frac{1}{1-q^{l}}+\mathcal{O}\left(q^{\left\lfloor\frac{d}{2}\right\rfloor+1}\right) .
$$

Equation (4.39): The proof is exactly as in Equation (4.38), except that $\mu \in P_{C}$ rather than $P_{R}$, and

$$
\sum_{\substack{\lambda \in P_{R} \\ \mu \in P_{C} \\ \lambda=\mu}} q^{\frac{|\lambda|}{2}}=\prod_{l=1}^{\infty} \frac{1}{1-q^{2 l}} .
$$

Equation (4.40): The proof is exactly as in Equation (4.39), except that $\lambda \in P_{C}$ and $\mu \in P_{R}$.

Equation (4.41): The proof is exactly as in Equation (4.38), except that $\lambda, \mu \in$ $P_{C}$.

Proof of Proposition 4.10. We will handle each case seperately.

Case $A_{R R}(q, \mathbf{x})$ :

$$
A_{R R}(q, \mathrm{x})=\frac{A_{R R}\left(q, q^{\frac{1}{2}} \mathrm{x}\right)}{\prod_{i \leq j}\left(1-q x_{i} x_{j}\right)} .
$$

Iterating 4.20) of Lemma 4.11 $d$ times, we obtain

$$
A_{R R}(q, \mathrm{x})=\frac{A_{R R}\left(q, q^{\frac{d}{2}} \mathrm{x}\right)}{\prod_{l=1}^{d} \prod_{i \leq j}\left(1-q^{l} x_{i} x_{j}\right)} .
$$

As a formal series in $q$ we have that, up to order $\lfloor d\rfloor$ in $q$,

$$
A_{R R}(q, \mathrm{x})=\frac{A_{R R}\left(q, q^{\frac{d}{2}} \mathrm{x}\right)}{\prod_{l=1}^{\infty} \prod_{i \leq j}\left(1-q^{l} x_{i} x_{j}\right)}+O\left(q^{d+1}\right) .
$$


We apply (4.38) of Lemma4.12 to obtain

$$
A_{R R}(q, \mathrm{x})=\frac{\prod_{l=1}^{\infty}\left(\frac{1}{1-q^{l}}\right)}{\prod_{l=1}^{\infty} \prod_{i \leq j}\left(1-q^{l} x_{i} x_{j}\right)}+O\left(q^{\left\lfloor\frac{d}{2}\right\rfloor+1}\right) .
$$

Notice that the left side of the above equation is independent of $d$, for all $d \geq 0$. The result follows.

Case $A_{R C}(q, \mathbf{x})$ : From (4.21) of Lemma 4.11, we have

$$
A_{R C}(q, \mathrm{x})=\frac{A_{C R}\left(q, q^{\frac{1}{2}} \mathrm{x}\right)}{\prod_{i \leq j}\left(1-q x_{i} x_{j}\right)} .
$$

Applying (4.22) of Lemma 4.11, we obtain

$$
A_{R C}(q, \mathrm{x})=\frac{A_{R C}(q, q \mathrm{x})}{\prod_{i \leq j}\left(1-q x_{i} x_{j}\right) \prod_{i<j}\left(1-q^{2} x_{i} x_{j}\right)} .
$$

Iterating the above 2 steps $d$ times, we find that

$$
\frac{A_{R C}\left(q, q^{\frac{2 d}{2}} \mathrm{x}\right)}{\prod_{l=1}^{d} \prod_{i \leq j}\left(1-q^{2 l-1} x_{i} x_{j}\right) \prod_{i<j}\left(1-q^{2 l} x_{i} x_{j}\right)} .
$$

Applying (4.39) of Lemma 4.12 we find that, as a formal series in $q, A_{R C}(q, \mathrm{x})$ is

$$
\frac{\prod_{l=1}^{\infty} \frac{1}{1-q^{2 l}}}{\prod_{l=1}^{\infty} \prod_{i \leq j}\left(1-q^{2 l-1} x_{i} x_{j}\right) \prod_{i<j}\left(1-q^{2 l} x_{i} x_{j}\right)}+O\left(q^{d+1}\right) .
$$

As before, the result follows from the fact that the left side is independent of $d$.

Case $A_{C R}(q, \mathbf{x})$ : This case is similar to that of $A_{R C}(q, \mathrm{x})$ with the roles of $R$ and $C$ interchanged, and Equations (4.22) and (4.21) from Lemma 4.11 interchanged. Also, in Lemma 4.12, use (4.40) instead of (4.39).

Case $A_{C C}(q, \mathbf{x})$ : Proceeding in a similar manner as in the $A_{R R}(q, \mathrm{x})$ case, we first establish from(4.23) of Lemma 4.11 that

$$
A_{C C}(q, \mathrm{x})=\frac{A_{C C}\left(q, q^{\frac{d}{2}} \mathrm{x}\right)}{\prod_{l=1}^{\infty} \prod_{i<j}\left(1-q^{l} x_{i} x_{j}\right)}+O\left(q^{d+1}\right) .
$$

Apply (4.41) of Lemma 4.12, to obtain

$$
A_{C C}(q, \mathrm{x})=\frac{\prod_{l=1}^{\infty}\left(\frac{1}{1-q^{l}}\right)}{\prod_{l=1}^{\infty} \prod_{i<j}\left(1-q^{l} x_{i} x_{j}\right)}+O\left(q^{\left\lfloor\frac{d}{2}\right\rfloor+1}\right) .
$$

Notice that the left side of the above equation is independent of $d$.

\section{The Stable Range}

We now prove Theorem 1.7 on a case by case basis. They are all similar, but not exactly the same. Consequently, case RR is done in detail, the others in less detail. Changes from the RR case will be pointed out when necessary. 


\subsection{Case RR.}

Proposition 5.1. For all partitions $\nu$,

$$
\frac{\widetilde{p}_{R R}(\nu, q)}{\prod_{l=1}^{\infty}\left(1-q^{l}\right)}=\sum_{\lambda, \mu \in P_{R}} c_{\mu \nu}^{\lambda} q^{\frac{|\lambda|}{2}}
$$

Proof. By (1.8) in Definition 1.5, we have

$$
\sum_{\nu} \frac{\widetilde{p}_{R R}(\nu, q)}{\prod_{l=1}^{\infty}\left(1-q^{l}\right)} s_{\nu}(\mathrm{x})=\prod_{l=1}^{\infty} \frac{1}{1-q^{l}}\left(\prod_{i \leq j} \frac{1}{1-q^{l} x_{i} x_{j}}\right) .
$$

By Proposition 4.10 the above expression is equal to

$$
\sum_{\lambda, \mu \in P_{R}} s_{\lambda / \mu}(\mathrm{x}) q^{\frac{|\lambda|}{2}}
$$

Applying Definition 4.5

$$
\sum_{\lambda, \mu \in P_{R}} \sum_{\nu} c_{\mu \nu}^{\lambda} s_{\nu}(\mathrm{x}) q^{\frac{|\lambda|}{2}}
$$

After rearranging the order of summation we obtain the result:

$$
\sum_{\nu}\left(\sum_{\lambda, \mu \in P_{R}} c_{\mu \nu}^{\lambda} q^{\frac{|\lambda|}{2}}\right) s_{\nu}(\mathrm{x})
$$

Let $p_{\nu}^{(n)}(q)$ be the $q$-multiplicity in the space $\mathcal{H}_{\mathfrak{p}}$ for the symmetric pair $(G L(n)$, $O(n))$. Then,

\section{Proposition 5.2.}

$$
\frac{p_{\nu}^{(n)}(q)}{\prod_{l=1}^{n}\left(1-q^{l}\right)}=\sum_{\substack{l(\lambda) \leq \frac{n}{2} \\ \lambda, \mu \in P_{R}}} c_{\mu \nu}^{\lambda} q^{\frac{|\lambda|}{2}}+\mathcal{O}\left(q^{\left\lfloor\frac{n}{2}\right\rfloor+1}\right)
$$

Proof. From the Kostant-Rallis theorem we have that the left side of (5.6) is the $q$-multiplicity of the $O(n)$ representation $E^{\nu}$ in the graded space $\mathcal{P}\left(S^{2} \mathbb{C}^{n}\right)$. We will expand the $q$-character of this space in another way to obtain the result. First we will decompose the space with respect to the action of $G L(n)$ from Proposition 2.4

$$
\operatorname{char}_{q} \mathcal{P}\left(S^{2} \mathbb{C}^{n}\right)=\sum_{\substack{\lambda \in P_{R} \\ l(\lambda) \leq n}} \operatorname{char} F_{(n)}^{\lambda} q^{\frac{|\lambda|}{2}} .
$$

Restricting the action to $O(n)$, we obtain

$$
\operatorname{char}_{q} \mathcal{P}\left(S^{2} \mathbb{C}^{n}\right)=\sum_{\substack{\lambda \in P_{R} \\ l(\lambda) \leq n}}\left(\sum_{\nu} C_{\nu}^{\lambda} \operatorname{char} E_{(n)}^{\nu}\right) q^{\frac{|\lambda|}{2}}
$$

We split off the terms in the above sum with $\lambda$ of size $n$ or smaller:

$$
\sum_{\substack{\lambda \in P_{R} \\|\lambda| \leq n, l(\lambda) \leq n}}\left(\sum_{\nu} C_{\nu}^{\lambda} \operatorname{char} E_{(n)}^{\nu}\right) q^{\frac{|\lambda|}{2}}
$$




$$
+\sum_{\substack{\lambda \in P_{R} \\|\lambda|>n, l(\lambda) \leq n}}\left(\sum_{\nu} C_{\nu}^{\lambda} \operatorname{char} E_{(n)}^{\nu}\right) q^{\frac{|\lambda|}{2}}
$$

Now we have an expression for the $q$-character up to order $\left\lfloor\frac{n}{2}\right\rfloor$ :

$$
\sum_{\substack{\lambda \in P_{R} \\ l(\lambda) \leq n}}\left(\sum_{\nu} C_{\nu}^{\lambda} \operatorname{char} E_{(n)}^{\nu}\right) q^{\frac{|\lambda|}{2}}+O\left(q^{\left\lfloor\frac{n}{2}\right\rfloor+1}\right) .
$$

Notice that if $\lambda \in P_{R}$, then $l(\lambda) \leq \frac{1}{2}|\lambda|$. This is because, if $\lambda \in P_{R}$, then $\lambda=2 \sigma$ for some partition $\sigma$. We have $l(\lambda)=l(\sigma), l(\sigma) \leq|\sigma|$, and $|\sigma|=\frac{1}{2}|\lambda|$. So if $|\lambda| \leq n$ and $\lambda \in P_{R}$, then $l(\lambda) \leq \frac{n}{2}$. Therefore $\operatorname{char}_{q} \mathcal{P}\left(S^{2} \mathbb{C}^{n}\right)$ is

$$
\sum_{\substack{\lambda \in P_{R} \\ l(\lambda) \leq \frac{n}{2}}}\left(\sum_{\nu} C_{\nu}^{\lambda} \operatorname{char} E_{(n)}^{\nu}\right) q^{\frac{|\lambda|}{2}}+O\left(q^{\left\lfloor\frac{n}{2}\right\rfloor+1}\right) .
$$

We can now apply Theorem 3.5 .

$$
\sum_{\substack{\lambda \in P_{R_{n}} \\ l(\lambda) \leq \frac{n}{2}}}\left(\sum_{\nu} \sum_{\mu \in P_{R}} c_{\mu \nu}^{\lambda} \operatorname{char} E_{(n)}^{\nu}\right) q^{\frac{|\lambda|}{2}}+O\left(q^{\left\lfloor\frac{n}{2}\right\rfloor+1}\right) .
$$

Extracting the coefficient of $\operatorname{char} E_{(n)}^{\nu}$ in the above expression, we obtain the right side of (5.66).

Proof of (1.18) of Theorem 1.7. The result is immediate from the fact that the right sides of (5.1) and (5.6) are equal up to order $\left\lfloor\frac{n}{2}\right\rfloor+1$ in $q$.

\subsection{Case RC.}

Proposition 5.3. For all partitions $\nu$,

$$
\frac{\widetilde{p}_{R C}(\nu, q)}{\prod_{l=1}^{\infty}\left(1-q^{2 l}\right)}=\sum_{\substack{\lambda \in P_{R} \\ \mu \in P_{C}}} c_{\mu \nu}^{\lambda} q^{\frac{|\lambda|}{2}} .
$$

Proof. By (1.9) in Definition 1.5 we have

$$
\begin{aligned}
& \sum_{\nu} \frac{\widetilde{p}_{R C}(\nu, q)}{\prod_{l=1}^{\infty}\left(1-q^{2 l}\right)} s_{\nu}(\mathrm{x}) \\
& =\prod_{l=1}^{\infty} \frac{1}{1-q^{2 l}}\left(\prod_{i \leq j} \frac{1}{1-q^{2 l-1} x_{i} x_{j}} \prod_{i<j} \frac{1}{1-q^{2 l} x_{i} x_{j}}\right) .
\end{aligned}
$$

By Proposition 4.10 the above expression is equal to

$$
\sum_{\substack{\lambda \in P_{R} \\ \mu \in P_{C}}} s_{\lambda / \mu}(\mathrm{x}) q^{\frac{|\lambda|}{2}}
$$

Applying Definition 4.5.

$$
\sum_{\substack{\lambda \in P_{R} \\ \mu \in P_{C}}} \sum_{\nu} c_{\mu \nu}^{\lambda} s_{\nu}(\mathrm{x}) q^{\frac{|\lambda|}{2}}
$$


After rearranging the order of summation we obtain the result:

$$
\sum_{\nu}\left(\sum_{\substack{\lambda \in P_{R} \\ \mu \in P_{C}}} c_{\mu \nu}^{\lambda} q^{\frac{|\lambda|}{2}}\right) s_{\nu}(\mathrm{x})
$$

Let $p_{\nu}^{(k)}(q)$ be the $q$-multiplicity in the space $\mathcal{H}_{\mathfrak{k}}$ for the $(G L(2 k), S p(k))$ case of Kostant's theorem.

\section{Proposition 5.4.}

$$
\frac{p_{\nu}^{(k)}(q)}{\prod_{l=1}^{k}\left(1-q^{2 l}\right)}=\sum_{\substack{l(\lambda) \leq k \\ \lambda \in P_{R}, \mu \in P_{C}}} c_{\mu \nu}^{\lambda} q^{\frac{|\lambda|}{2}}+\mathcal{O}\left(q^{k+1}\right) .
$$

Proof. From Kostant's theorem we have that the left side of (5.18) is the $q$ multiplicity of the $S p(k)$ representation $V^{\nu}$ in the graded space $\mathcal{P}\left(S^{2} \mathbb{C}^{2 k}\right)$. We will expand the $q$-character of this space in another way to obtain the result. First we will decompose the space with respect to the action of $G L(2 k)$ from Proposition 2.4 .

$$
\operatorname{char}_{q} \mathcal{P}\left(S^{2} \mathbb{C}^{2 k}\right)=\sum_{\substack{\lambda \in P_{R} \\ l(\lambda) \leq 2 k}} \operatorname{char} F_{(2 k)}^{\lambda} q^{\frac{|\lambda|}{2}} .
$$

We then restrict the action to $S p(k)$ and expand in terms of the irreducible characters for the subgroup:

$$
\operatorname{char}_{q} \mathcal{P}\left(S^{2} \mathbb{C}^{2 k}\right)=\sum_{\substack{\lambda \in P_{R} \\ l(\lambda) \leq 2 k}}\left(\sum_{\nu} D_{\nu}^{\lambda} \operatorname{char} V_{(k)}^{\nu}\right) q^{\frac{|\lambda|}{2}} .
$$

We split off the terms in the above sum with $\lambda$ of size $k$ or smaller:

$$
\begin{aligned}
& \sum_{\substack{\lambda \in P_{R} \\
|\lambda| \leq 2 k, l(\lambda) \leq 2 k}}\left(\sum_{\nu} D_{\nu}^{\lambda} \operatorname{char} V_{(k)}^{\nu}\right) q^{\frac{|\lambda|}{2}} \\
+ & \sum_{\substack{\lambda \in P_{R} \\
|\lambda|>2 k, l(\lambda) \leq 2 k}}\left(\sum_{\nu} D_{\nu}^{\lambda} \operatorname{char} V_{(k)}^{\nu}\right) q^{\frac{|\lambda|}{2}} .
\end{aligned}
$$

Now we have an expression for the $q$-character up to order $k$ in $q$ :

$$
\sum_{\substack{\lambda \in P_{R} \\ l(\lambda) \leq k}}\left(\sum_{\nu} D_{\nu}^{\lambda} \operatorname{char} V_{(k)}^{\nu}\right) q^{\frac{|\lambda|}{2}}+\mathcal{O}\left(q^{k+1}\right) .
$$

Since $\lambda \in P_{R}$, we have $l(\lambda) \leq \frac{1}{2}|\lambda|$ and so $l(\lambda) \leq k$. Therefore,

$$
\operatorname{char}_{q} \mathcal{P}\left(S^{2} \mathbb{C}^{2 k}\right)=\sum_{\substack{\lambda \in P_{R} \\ l(\lambda) \leq k}}\left(\sum_{\nu} D_{\nu}^{\lambda} \operatorname{char} V_{(k)}^{\nu}\right) q^{\frac{|\lambda|}{2}}+\mathcal{O}\left(q^{k+1}\right) .
$$


We can now apply Theorem 3.7 ;

$$
\sum_{\substack{\lambda \in P_{R} \\ l(\lambda) \leq k}}\left(\sum_{\nu} \sum_{\mu \in P_{C}} c_{\mu \nu}^{\lambda} \operatorname{char} V_{(k)}^{\nu}\right) q^{\frac{|\lambda|}{2}}+O\left(q^{k+1}\right) .
$$

Extracting the coefficient of $\operatorname{char} V_{(k)}^{\nu}$ in the above expression, we obtain the right side of Equation (5.18).

Proof of (1.19) in Theorem 1.7. The result is immediate from the fact that the right sides of (5.13) and (5.18) are equal up to order $k$ in $q$.

\subsection{Case CR.}

Proposition 5.5. For all partitions $\nu$,

$$
\frac{\widetilde{p}_{C R}(\nu, q)}{\prod_{l=1}^{\infty}\left(1-q^{2 l}\right)}=\sum_{\substack{\lambda \in P_{C} \\ \mu \in P_{R}}} c_{\mu \nu}^{\lambda} q^{\frac{|\lambda|}{2}} .
$$

Proof. By (1.10) of Definition 1.5, we have

$$
\begin{aligned}
& \sum_{\nu} \frac{\widetilde{p}_{C R}(\nu, q)}{\prod_{l=1}^{\infty}\left(1-q^{2 l}\right)} s_{\nu}(\mathrm{x}) \\
& =\prod_{l=1}^{\infty} \frac{1}{1-q^{2 l}}\left(\prod_{i \leq j} \frac{1}{1-q^{2 l} x_{i} x_{j}} \prod_{i<j} \frac{1}{1-q^{2 l-1} x_{i} x_{j}}\right) .
\end{aligned}
$$

By Proposition 4.10 the above expression is equal to

$$
\sum_{\substack{\lambda \in P_{C} \\ \mu \in P_{R}}} s_{\lambda / \mu}(\mathrm{x}) q^{\frac{|\lambda|}{2}}
$$

Applying Definition 4.5

$$
\sum_{\substack{\lambda \in P_{C} \\ \mu \in P_{R}}} \sum_{\nu} c_{\mu \nu}^{\lambda} s_{\nu}(\mathrm{x}) q^{\frac{|\lambda|}{2}} .
$$

After rearranging the order of summation we obtain the result:

$$
\sum_{\nu}\left(\sum_{\substack{\lambda \in P_{C} \\ \mu \in P_{R}}} c_{\mu \nu}^{\lambda} q^{\frac{|\lambda|}{2}}\right) s_{\nu}(\mathrm{x})
$$

Remark 5.6. For $\mathfrak{p} \cong \wedge^{2}\left(\mathbb{C}^{n}\right)$ and $K=O(n)$, we have that $\mathcal{P}(\mathfrak{p})$ is free over $\mathcal{P}(\mathfrak{p})^{K}$. This does not follow directly from Kostant's theorem since $K$ is not connected. However, we can reduce the freeness assertion to the case of $S O(n)$.

Let $p_{\nu}^{(n)}(q)$ be the $q$-multiplicity in the space $\mathcal{H}_{\mathfrak{k}}$ for the symmetric pair $(G L(n)$, $O(n))$.

Proposition 5.7.

$$
\frac{p_{\nu}^{(n)}(q)}{\prod_{l=1}^{\left\lfloor\frac{n}{2}\right\rfloor}\left(1-q^{2 l}\right)}=\sum_{\substack{l(\lambda) \leq n \\ \lambda \in P_{C}} \in P_{R}} c_{\mu \nu}^{\lambda} q^{\frac{|\lambda|}{2}}+\mathcal{O}\left(q^{\left\lfloor\frac{n}{4}\right\rfloor+1}\right) .
$$


Proof. By the above remark, the left side of (5.30) is the $q$-multiplicity of the $O(n)$ representation $E^{\nu}$ in the graded space $\mathcal{P}\left(\wedge^{2} \mathbb{C}^{n}\right)$. We will expand the $q$-character of this space in another way to obtain the result. First we decompose the space with respect to the action of $G L(n)$ from Proposition 2.5

$$
\operatorname{char}_{q} \mathcal{P}\left(\wedge^{2} \mathbb{C}^{n}\right)=\sum_{\substack{\lambda \in P_{C} \\ l(\lambda) \leq n}} \operatorname{char} F_{(n)}^{\lambda} q^{\frac{|\lambda|}{2}}
$$

We then restrict the action to $O(n)$ and expand in terms of the irreducible characters for the subgroup:

$$
\operatorname{char}_{q} \mathcal{P}\left(\wedge^{2} \mathbb{C}^{n}\right)=\sum_{\substack{\lambda \in P_{C} \\ l(\lambda) \leq n}}\left(\sum_{\nu} C_{\nu}^{\lambda} \operatorname{char} E_{(n)}^{\nu}\right) q^{\frac{|\lambda|}{2}} .
$$

We split off the terms in the above sum that correspond to those $\lambda$ of size $\left\lfloor\frac{n}{2}\right\rfloor$ or smaller:

$$
\begin{aligned}
& \sum_{\substack{\lambda \in P_{C} \\
|\lambda| \leq\left\lfloor\frac{n}{2}\right\rfloor, l(\lambda) \leq n}}\left(\sum_{\nu} C_{\nu}^{\lambda} \operatorname{char} E_{(n)}^{\nu}\right) q^{\frac{|\lambda|}{2}} \\
&+\sum_{\substack{\lambda \in P_{C} \\
|\lambda|>\left\lfloor\frac{n}{2}\right\rfloor, l(\lambda) \leq n}}\left(\sum_{\nu} C_{\nu}^{\lambda} \operatorname{char} E_{(n)}^{\nu}\right) q^{\frac{|\lambda|}{2}} .
\end{aligned}
$$

Now we have an expression for the $q$-character up to order $\left\lfloor\frac{n}{4}\right\rfloor$ :

$$
\sum_{\substack{\lambda \in P_{C} \\ l(\lambda) \leq n}}\left(\sum_{\nu} C_{\nu}^{\lambda} \operatorname{char} E_{(n)}^{\nu}\right) q^{\frac{|\lambda|}{2}}+O\left(q^{\left\lfloor\frac{n}{4}\right\rfloor+1}\right) .
$$

If $|\lambda| \leq\left\lfloor\frac{n}{2}\right\rfloor$, then $l(\lambda) \leq\left\lfloor\frac{n}{2}\right\rfloor$. Therefore,

$$
\operatorname{char}_{q} \mathcal{P}\left(\wedge^{2} \mathbb{C}^{n}\right)=\sum_{\substack{\lambda \in P_{C} \\ l(\lambda) \leq\left\lfloor\frac{n}{2}\right\rfloor}}\left(\sum_{\nu} C_{\nu}^{\lambda} \operatorname{char} E_{(n)}^{\nu}\right) q^{\frac{|\lambda|}{2}}+O\left(q^{\left\lfloor\frac{n}{4}\right\rfloor+1}\right) .
$$

We can now apply Theorem 3.5 .

$$
\sum_{\substack{\lambda \in P_{C} \\ l(\lambda) \leq\left\lfloor\frac{n}{2}\right\rfloor}}\left(\sum_{\nu} \sum_{\mu \in P_{R}} c_{\mu \nu}^{\lambda} \operatorname{char} E_{(n)}^{\nu}\right) q^{\frac{|\lambda|}{2}}+O\left(q^{\left\lfloor\frac{n}{4}\right\rfloor+1}\right) .
$$

Extracting the coefficient of $\operatorname{char} E_{(n)}^{\nu}$ in the above expression, we obtain the right side of (5.30).

Proof of (1.20) of Theorem 1.7. The result is immediate from the fact that the right sides of (5.25) and (5.30) are equal up to order $\left\lfloor\frac{n}{4}\right\rfloor$ in $q$. 


\subsection{Case CC.}

Proposition 5.8. For all partitions $\nu$,

$$
\frac{\tilde{p}_{C C}(\nu, q)}{\prod_{l=1}^{\infty}\left(1-q^{l}\right)}=\sum_{\lambda, \mu \in P_{C}} c_{\mu \nu}^{\lambda} q^{\frac{|\lambda|}{2}}
$$

Proof. By 1.11 in Definition 1.5, we have

$$
\sum_{\nu} \frac{\tilde{p}_{C C}(\nu, q)}{\prod_{l=1}^{\infty}\left(1-q^{l}\right)} s_{\nu}(\mathrm{x})=\prod_{l=1}^{\infty} \frac{1}{1-q^{l}}\left(\prod_{i<j} \frac{1}{1-q^{l} x_{i} x_{j}}\right) .
$$

By Proposition 4.10 the above expression is equal to

$$
\sum_{\lambda, \mu \in P_{C}} s_{\lambda / \mu}(\mathrm{x}) q^{\frac{|\lambda|}{2}} .
$$

Applying Definition 4.5

$$
\sum_{\lambda, \mu \in P_{C}} \sum_{\nu} c_{\mu \nu}^{\lambda} s_{\nu}(\mathrm{x}) q^{\frac{|\lambda|}{2}}
$$

After rearranging the order of summation we obtain the result:

$$
\sum_{\nu}\left(\sum_{\lambda, \mu \in P_{C}} c_{\mu \nu}^{\lambda} q^{\frac{|\lambda|}{2}}\right) s_{\nu}(\mathrm{x})
$$

Let $p_{\nu}^{(k)}(q)$ be the $q$-multiplicity in the space $\mathcal{H}_{\mathfrak{p}}$ for the symmetric pair $(G L(2 k)$, $S p(k))$.

\section{Proposition 5.9.}

$$
\frac{p_{\nu}^{(n)}(q)}{\prod_{l=1}^{k}\left(1-q^{l}\right)}=\sum_{\substack{l(\lambda) \leq k \\ \lambda, \mu \in P_{C}}} c_{\mu \nu}^{\lambda} q^{\frac{|\lambda|}{2}}+\mathcal{O}\left(q^{\left\lfloor\frac{k}{2}\right\rfloor+1}\right)
$$

Proof. By the Kostant-Rallis theorem the left side of (5.42) is the $q$-multiplicity of the $S p(k)$ representation $V^{\nu}$ in the graded space $\mathcal{P}\left(\wedge^{2}\left(\mathbb{C}^{2 k}\right)\right)$. We will expand the $q$-character of this space in another way to obtain the result. First we decompose the space with respect to the action of $G L(2 k)$ from Proposition 2.4:

$$
\operatorname{char}_{q} \mathcal{P}\left(\wedge^{2} \mathbb{C}^{2 k}\right)=\sum_{\substack{\lambda \in P_{C} \\ l(\lambda) \leq 2 k}} \operatorname{char} F_{(2 k)}^{\lambda} q^{\frac{|\lambda|}{2}} .
$$

We then restrict the action to $S p(k)$ :

$$
\operatorname{char}_{q} \mathcal{P}\left(\wedge^{2} \mathbb{C}^{2 k}\right)=\sum_{\substack{\lambda \in P_{C} \\ l(\lambda) \leq 2 k}}\left(\sum_{\nu} D_{\nu}^{\lambda} \operatorname{char} V_{(k)}^{\nu}\right) q^{\frac{|\lambda|}{2}} .
$$

We split off the terms in the above sum with $\lambda$ of size $k$ or smaller:

$$
\sum_{\substack{\lambda \in P_{C} \\|\lambda| \leq k, l(\lambda) \leq 2 k}}\left(\sum_{\nu} D_{\nu}^{\lambda} \operatorname{char} V_{(k)}^{\nu}\right) q^{\frac{|\lambda|}{2}}
$$




$$
+\sum_{\substack{\lambda \in P_{C} \\|\lambda|>k, l(\lambda) \leq 2 k}}\left(\sum_{\nu} D_{\nu}^{\lambda} \operatorname{char} V_{(k)}^{\nu}\right) q^{\frac{|\lambda|}{2}}
$$

Now we have an expression for the $q$-character up to order $\left\lfloor\frac{k}{2}\right\rfloor$ :

$$
\sum_{\substack{\lambda \in P_{C} \\ l(\lambda) \leq 2 k}}\left(\sum_{\nu} D_{\nu}^{\lambda} \operatorname{char} V_{(k)}^{\nu}\right) q^{\frac{|\lambda|}{2}}+O\left(q^{\left\lfloor\frac{k}{2}\right\rfloor+1}\right) .
$$

But $l(\lambda) \leq|\lambda|$; so we have $l(\lambda) \leq k$ in the above expression. Therefore,

$$
\operatorname{char}_{q} \mathcal{P}\left(\wedge^{2} \mathbb{C}^{n}\right)=\sum_{\substack{\lambda \in P_{C} \\ l(\lambda) \leq k}}\left(\sum_{\nu} D_{\nu}^{\lambda} \operatorname{char} E_{(n)}^{\nu}\right) q^{\frac{|\lambda|}{2}}+O\left(q^{\left\lfloor\frac{k}{2}\right\rfloor+1}\right) .
$$

We can now apply Theorem 3.7 ;

$$
\sum_{\substack{\lambda \in P_{C} \\ l(\lambda) \leq k}}\left(\sum_{\nu} \sum_{\mu \in P_{C}} c_{\mu \nu}^{\lambda} \operatorname{char} V_{(k)}^{\nu}\right) q^{\frac{|\lambda|}{2}}+O\left(q^{\left\lfloor\frac{k}{2}\right\rfloor+1}\right) .
$$

Extracting the coefficient of $\operatorname{char} V_{(k)}^{\nu}$ in the above expression, we obtain the right side of (5.42).

Proof of (1.21) of Theorem 1.7. The result is immediate from the fact that the right sides of (5.37) and (5.42) are equal up to order $\left\lfloor\frac{k}{2}\right\rfloor$ in $q$.

\section{GR GR AND $G C G C$}

Proposition 6.1. The Hilbert series is

$$
\prod_{l=1}^{\min (k, n)}\left(\frac{1}{1-q^{2 l}}\right)
$$

for $\mathcal{P}\left(M_{k \times n}\right)^{O(k) \times O(n)}$ or for $\mathcal{P}\left(M_{2 k \times 2 n}\right)^{S p(k) \times S p(n)}$.

Proof. Using Proposition 2.3 and Theorem 2.6, the Hilbert series for $O(k) \times O(n)$ is seen to be

$$
\sum_{\substack{\lambda \in P_{R} \\ l(\lambda) \leq \min (k, n)}} q^{|\lambda|}
$$

and for $S p(k) \times S p(n)$,

$$
\sum_{\substack{\lambda \in P_{C} \\ l(\lambda) \leq \min (2 k, 2 n)}} q^{|\lambda|}
$$

Each formal sum can be easily closed to obtain the stated rational expressions.

\section{Proposition 6.2.}

$$
\sum_{\lambda} s_{\lambda / \alpha}(\mathrm{x}) s_{\lambda / \beta}(\mathrm{y})=\prod_{i, j} \frac{1}{1-x_{i} y_{j}} \sum_{\mu} s_{\alpha / \mu}(\mathrm{x}) s_{\beta / \mu}(\mathrm{y})
$$


Proof (from [8], p. 93 (Chapter I, Section 5, Example 26)). We have

$$
R(\mathrm{x}, \mathrm{y}, \mathrm{z}, \mathrm{w}):=\prod_{i, j}\left(\frac{1}{1-x_{i} y_{j}} \frac{1}{1-x_{i} w_{j}} \frac{1}{1-z_{i} y_{j}} \frac{1}{1-z_{i} w_{j}}\right) .
$$

From Proposition 4.1 the above is

$$
\sum_{\lambda} s_{\lambda}(\mathrm{x}, \mathrm{z}) s_{\lambda}(\mathrm{y}, \mathrm{w}) .
$$

Using Proposition 4.6.

$$
R(\mathrm{x}, \mathrm{y}, \mathrm{z}, \mathrm{w})=\sum_{\lambda, \alpha, \beta} s_{\lambda / \alpha}(\mathrm{x}) s_{\lambda / \beta}(\mathrm{y}) s_{\alpha}(\mathrm{z}) s_{\beta}(\mathrm{w}) .
$$

Now expand $R(\mathrm{x}, \mathrm{y}, \mathrm{z}, \mathrm{w})$ as follows:

$$
\begin{aligned}
& \prod_{i, j} \frac{1}{1-x_{i} y_{j}} \sum_{\lambda, \tau, \sigma} s_{\sigma}(\mathrm{x}) s_{\sigma}(\mathrm{w}) s_{\tau}(\mathrm{z}) s_{\tau}(\mathrm{y}) s_{\lambda}(\mathrm{z}) s_{\lambda}(\mathrm{w}) \\
& =\prod_{i, j} \frac{1}{1-x_{i} y_{j}} \sum_{\lambda, \alpha, \beta, \tau, \sigma} c_{\lambda \sigma}^{\beta} c_{\lambda \tau}^{\alpha} s_{\tau}(\mathrm{x}) s_{\sigma}(\mathrm{y}) s_{\alpha}(\mathrm{z}) s_{\beta}(\mathrm{w}) \\
& =\prod_{i, j} \frac{1}{1-x_{i} y_{j}} \sum_{\lambda, \alpha, \beta} s_{\alpha / \lambda}(\mathrm{x}) s_{\beta / \lambda}(\mathrm{y}) s_{\alpha}(\mathrm{z}) s_{\beta}(\mathrm{w}) .
\end{aligned}
$$

Now take the coefficient of $s_{\alpha}(\mathrm{z}) s_{\beta}(\mathrm{w})$ in (6.6) and 6.9).

Definition 6.3.

$$
\begin{aligned}
& A_{G R, G R}(q, \mathrm{x}, \mathrm{y}):=\sum_{\lambda} \sum_{\mu, \nu \in P_{R}} s_{\lambda / \mu}(\mathrm{x}) s_{\lambda / \nu}(\mathrm{y}) q^{|\lambda|}, \\
& A_{G C, G C}(q, \mathrm{x}, \mathrm{y}):=\sum_{\lambda} \sum_{\mu, \nu \in P_{C}} s_{\lambda / \mu}(\mathrm{x}) s_{\lambda / \nu}(\mathrm{y}) q^{|\lambda|} .
\end{aligned}
$$

\section{Proposition 6.4.}

$$
A_{G R, G R}(q, \mathrm{x}, \mathrm{y})=\frac{A_{G R, G R}(q, q \mathrm{x}, q \mathrm{y})}{\prod_{i, j}\left(1-q x_{i} y_{j}\right) \prod_{i \leq j}\left(1-q^{2} x_{i} x_{j}\right)\left(1-q^{2} y_{i} y_{j}\right)}
$$

and

$$
A_{G C, G C}(q, \mathrm{x}, \mathrm{y})=\frac{A_{G C, G C}(q, q \mathrm{x}, q \mathrm{y})}{\prod_{i, j}\left(1-q x_{i} y_{j}\right) \prod_{i<j}\left(1-q^{2} x_{i} x_{j}\right)\left(1-q^{2} y_{i} y_{j}\right)} .
$$

Proof. We prove the first case, as the second is similar. By definition $A_{G R, G R}(q, \mathrm{x}, \mathrm{y})$ is

$$
\sum_{\lambda} \sum_{\mu, \nu \in P_{R}} s_{\lambda / \mu}(\mathrm{x}) s_{\lambda / \nu}(\mathrm{y}) q^{|\lambda|} .
$$

Using homogeneity and Proposition 6.2

$$
\begin{aligned}
& A_{G R, G R}(q, \mathrm{x}, \mathrm{y})=\sum_{\lambda} \sum_{\mu, \nu \in P_{R}} s_{\lambda / \mu}\left(q^{\frac{1}{2}} \mathrm{x}\right) s_{\lambda / \nu}\left(q^{\frac{1}{2}} \mathrm{y}\right) q^{\frac{|\mu|+|\nu|}{2}} \\
& =\prod_{i, j} \frac{1}{1-q x_{i} y_{j}} \sum_{\lambda} \sum_{\mu, \nu \in P_{R}} s_{\mu / \lambda}\left(q^{\frac{1}{2}} \mathrm{x}\right) s_{\nu / \lambda}\left(q^{\frac{1}{2}} \mathrm{y}\right) q^{\frac{|\mu|+|\nu|}{2}}
\end{aligned}
$$




$$
\begin{aligned}
& =\prod_{i, j} \frac{1}{1-q x_{i} y_{j}} \sum_{\lambda} \sum_{\mu, \nu \in P_{R}} s_{\mu / \lambda}(q \mathrm{x}) s_{\nu / \lambda}(q \mathrm{y}) q^{|\lambda|} \\
& =\frac{\sum_{\lambda} \sum_{\mu, \nu \in P_{R}} s_{\lambda / \mu}(q \mathrm{x}) s_{\lambda / \nu}(q \mathrm{y}) q^{|\lambda|}}{\prod_{i, j}\left(1-q x_{i} y_{j}\right) \prod_{i \leq j}\left(1-q^{2} x_{i} x_{j}\right)\left(1-q^{2} y_{i} y_{j}\right)} .
\end{aligned}
$$

Proposition 6.5. As formal power series in $q$,

$$
A_{G R, G R}\left(q, q^{d} \mathrm{x}, q^{d} \mathrm{y}\right)=\prod_{l=1}^{\infty} \frac{1}{1-q^{2 l}}+\mathcal{O}\left(q^{2 d+1}\right)
$$

and

$$
A_{G C, G C}\left(q, q^{d} \mathrm{x}, q^{d} \mathrm{y}\right)=\prod_{l=1}^{\infty} \frac{1}{1-q^{2 l}}+\mathcal{O}\left(q^{2 d+1}\right) .
$$

Proof. We expand the expression defining $A_{G R, G R}$ as a series in $q$. The polynomials $s_{\lambda / \mu}(\mathrm{x})$ are homogeneous polynomials of degree $|\lambda|-|\mu|$. Hence,

$$
\begin{aligned}
A_{G R, G R}\left(q, q^{\frac{d}{2}} \mathrm{x}, q^{\frac{d}{2}} \mathrm{y}\right) & =\sum_{\substack{\mu, \nu \in P_{R} \\
\lambda}} s_{\lambda / \mu}\left(q^{\frac{d}{2}} \mathrm{x}\right) s_{\lambda / \nu}\left(q^{\frac{d}{2}} \mathrm{y}\right) q^{|\lambda|} \\
& =\sum_{\substack{\mu, \nu \in P_{R} \\
\lambda}} s_{\lambda / \mu}(\mathrm{x}) s_{\lambda / \nu}(\mathrm{y}) q^{\frac{d}{2}(2|\lambda|-|\mu|-|\nu|)+|\lambda|} .
\end{aligned}
$$

Separate the sum into two cases: $|\lambda|=|\mu|=|\nu|$ and not. Thus,

$$
\begin{aligned}
& \sum_{\substack{\mu, \nu \in P_{R} \\
\lambda: \lambda=\mu=\nu}} s_{\lambda / \mu}(\mathrm{x}) s_{\lambda / \nu}(\mathrm{y}) q^{\frac{d}{2}(2|\lambda|-|\mu|-|\nu|)+|\lambda|} \\
+ & \sum_{\substack{\lambda, \mu \in P_{R} \\
\lambda: \lambda \neq \mu \text { or } \lambda \neq \nu}} s_{\lambda / \mu}(\mathrm{x}) s_{\lambda / \nu}(\mathrm{y}) q^{\frac{d}{2}(2|\lambda|-|\mu|-|\nu|)+|\lambda|} .
\end{aligned}
$$

If the defining partitions are the same, then the skew Schur function is identically $1 ;$ so the above expression simplifies as

$$
\begin{aligned}
& =\sum_{\substack{\mu, \nu \in P_{R} \\
\lambda: \lambda=\mu=\nu}} q^{\frac{d}{2}(2|\lambda|-|\mu|-|\nu|)+|\lambda|} \\
& +\sum_{\substack{\lambda, \mu \in P_{R} \\
\lambda: \lambda \neq \mu \text { or } \lambda \neq \nu}} s_{\lambda / \mu}(\mathrm{x}) s_{\lambda / \nu}(\mathrm{y}) q^{\frac{d}{2}(2|\lambda|-|\mu|-|\nu|)+|\lambda|} .
\end{aligned}
$$

The formal series in the first sum is easily seen to be

$$
\prod_{l=1}^{\infty} \frac{1}{1-q^{2 l}}
$$

The lowest power of $q$ occurring in the second term is obtained when $2|\lambda|-|\mu|-|\nu|=$

1. In this case, the power is not less than $\left\lfloor\frac{d}{2}\right\rfloor+1$. Hence, (6.23) is

$$
\prod_{l=1}^{\infty} \frac{1}{1-q^{2 l}}+\mathcal{O}\left(q^{\left\lfloor\frac{d}{2}\right\rfloor+1}\right)
$$


The proof for $A_{G C, G C}$ is similar.

\section{Proposition 6.6.}

$$
\begin{aligned}
& A_{G R, G R}(q, \mathrm{x}, \mathrm{y}) \\
& =\prod_{l=1}^{\infty}\left(\frac{1}{1-q^{2 l}} \prod_{i, j} \frac{1}{1-q^{2 l-1} x_{i} y_{j}} \prod_{i \leq j} \frac{1}{\left(1-q^{2 k} x_{i} x_{j}\right)\left(1-q^{2 k} y_{i} y_{j}\right)}\right), \\
& A_{G C, G C}(q, \mathrm{x}, \mathrm{y}) \\
& =\prod_{l=1}^{\infty}\left(\frac{1}{1-q^{2 l}} \prod_{i, j} \frac{1}{1-q^{2 l-1} x_{i} y_{j}} \prod_{i<j} \frac{1}{\left(1-q^{2 k} x_{i} x_{j}\right)\left(1-q^{2 k} y_{i} y_{j}\right)}\right) .
\end{aligned}
$$

Proof. Iterate Proposition $6.4 d$ times and then use Proposition 6.5 The proof of the assertion for $A_{G C, G C}(\mu, \nu, q)$ is similar.

\section{Proposition 6.7.}

$$
\begin{aligned}
& \frac{\widetilde{p}_{G R, G R}(\mu, \nu, q)}{\prod_{l=1}^{\infty}\left(1-q^{2 l}\right)}=\sum_{\substack{\sigma, \tau \in P_{R} \\
\lambda}} c_{\sigma \mu}^{\lambda} c_{\tau \nu}^{\lambda} q^{|\lambda|}, \\
& \frac{\widetilde{p}_{G C, G C}(\mu, \nu, q)}{\prod_{l=1}^{\infty}\left(1-q^{2 l}\right)}=\sum_{\substack{\sigma, \tau \in P_{C} \\
\lambda}} c_{\sigma \mu}^{\lambda} c_{\tau \nu}^{\lambda} q^{|\lambda|} .
\end{aligned}
$$

Proof. The result follows immediately by expanding the skew Schur functions in the definition of $A_{G R, G R}$ or $A_{G C, G C}$ and then matching the resulting expressions with those given in Proposition 6.6.

Set $G=O(k+n), K=O(k) \times O(n), G^{\prime}=S O(k+n)$ and $K^{\prime}=S(O(k) \times O(n)):=$ $K \cap S L(k+n)$. We can take $\mathfrak{p}$ to be the complex $k \times n$ matrices $M_{k, n}$ under the action $(g, h) \cdot X=g X h^{-1}$ for $g \in G L(k), h \in G L(n)$ and $X \in M_{k, n}$. This action defines an action of $K$ and $K^{\prime}$ on $\mathfrak{p}$ by restriction which is the same as the restriction of the adjoint action of $G$ on $\mathfrak{p} . \mathcal{P}(\mathfrak{p})$ is a free module over $\mathcal{P}(\mathfrak{p})^{K}$. This fact does not follow directly from the Kostant-Rallis theorem, because $G$ is disconnected. In the following, we reduce this freeness assertion to the $\left(G^{\prime}, K^{\prime}\right)$ case of the Kostant-Rallis theorem.

See [2], Chapter 12, Section 4, Example 6, for the following description of the invariant ring $\mathcal{P}(\mathfrak{p})^{K^{\prime}}$ : If $k \neq n$, then $\mathcal{P}(\mathfrak{p})^{K^{\prime}}$ is generated by the algebraically independent polynomials $u_{i}=\operatorname{Trace}\left(\left(X X^{T}\right)^{i}\right)$ for $i=1, \ldots, \min (k, n)$. If $n=k$, then $\mathcal{P}(\mathfrak{p})^{K^{\prime}}=\mathbb{C}\left[u_{1}, u_{2}, \cdots, u_{n-1}, p\right]$, where $p=\operatorname{det}(X)$, with $u_{1}, \cdots, u_{n-1}$, and $p$ algebraically independent.

We should remark that in the $\left(G^{\prime}, K^{\prime}\right)$ case of the Kostant-Rallis theorem, the Lie algebra $\mathfrak{g}^{\prime}$ of $G^{\prime}$ can be taken as the skew-symmetric matrices of size $(k+n) \times(k+n)$ with $X \in M_{k, n} \cong \mathfrak{p}$, embedded as

$$
v(X)=\left[\begin{array}{cc}
0 & X \\
-X^{T} & 0
\end{array}\right] \in \mathfrak{g}^{\prime} .
$$

Under this embedding the polynomial $p$ is the Pfaffian of $v(X)$. If $n=k$, then $X$ is in fact a square matrix and $p=\operatorname{det}(X)$. 
Proposition 6.8. The Hilbert series of $\mathcal{P}\left(M_{k, n}\right)^{S(O(k) \times O(n))}$ is

$$
\begin{cases}\prod_{l=1}^{\min (k, n)}\left(\frac{1}{1-q^{2 l}}\right) & \text { if } n \neq k, \\ \frac{1}{1-q^{n}} \prod_{l=1}^{n-1}\left(\frac{1}{1-q^{2 l}}\right) & \text { if } n=k .\end{cases}
$$

Proof. The result follows from the fact that the invariant ring is generated by the above algebraically independent polynomials having the appropriate degrees.

Proposition 6.9. $\mathcal{P}\left(M_{k, n}\right)$ is a free module over $\mathcal{P}\left(M_{k, n}\right)^{O(k) \times O(n)}$.

Proof. In general, if $R$ and $R^{\prime}$ are rings and $R \subseteq R^{\prime}$, then $R^{\prime}$ can be regarded as a module over $R$. If $M$ is a module over $R^{\prime}$, then $M$ may be regarded as a module for $R$. Furthermore, if $M$ is a free module over $R^{\prime}$ and $R^{\prime}$ is a free module over $R$, then it follows (from the definition of a free module) that $M$ is free over $R$.

We apply the above principle to the case where $R=\mathcal{P}(\mathfrak{p})^{K}, R^{\prime}=\mathcal{P}(\mathfrak{p})^{K^{\prime}}$ and $M=\mathcal{P}(\mathfrak{p})$. By the Kostant-Rallis theorem for the pair $\left(G^{\prime}, K^{\prime}\right), M$ is free over $R^{\prime}$. The result will follow if we show that $R^{\prime}$ is free over $R$. To do this we will look at a set of generators for $R$ and $R^{\prime}$.

If $n \neq k$, then $R=R^{\prime}$, because $R \subseteq R^{\prime}$ and they have the same Hilbert series (from Propositions 6.1 and 6.8). Clearly, $R^{\prime}$ is free over $R$ in this case.

If $n=k$, then $\mathcal{P}(\mathfrak{p})^{K^{\prime}}=\mathbb{C}\left[u_{1}, u_{2}, \cdots, u_{n-1}, p\right]$ with $u_{1}, \cdots, u_{n-1}, p$ algebraically independent. It follows that the polynomials $u_{1}, u_{2}, \cdots, u_{n-1}, p^{2}$ are algebraically independent as well. $u_{1}, \cdots, u_{n-1}$ are invariant under both $K$ and $K^{\prime} . p$ is invariant under $K^{\prime}$ but not $K$; however, $p^{2}$ is invariant under $K$ (and $K^{\prime}$ ). Therefore, $R=$ $\mathbb{C}\left[u_{1}, \cdots, u_{n-1}, p^{2}\right]$, because $\left\{u_{1}, \cdots, u_{n-1}, p^{2}\right\}$ is contained in $R$ and the Hilbert series of $R$ (see Propositions 6.1) is the same as the Hilbert series of the polynomial ring generated by $\left\{u_{1}, \cdots, u_{n-1}, p^{2}\right\}$.

It remains to observe that the ring $R^{\prime}=\mathbb{C}\left[u_{1}, \cdots, u_{n-1}, p\right]$ is a free module over the subring $R=\mathbb{C}\left[u_{1}, \cdots, u_{n-1}, p^{2}\right]$. It is easy to see that $\{1, p\}$ is an $R$-basis for $R^{\prime}$, since the generators $u_{1}, \cdots, u_{n-1}, p$ are algebraically independent.

Proceeding as in the proofs of Propositions [5.2, 5.4 5.7 and 5.9] we obtain the next two propositions.

Proposition 6.10. Let $p_{\mu, \nu}^{(k, n)}(q)$ be the $q-$ multiplicity of $E_{(k)}^{\mu} \otimes E_{(n)}^{\nu}$ in the space $\mathcal{H}_{\mathfrak{p}}$ for the symmetric pair $(O(k+n), O(k) \times O(n))$. Then,

$$
\frac{p_{\mu, \nu}^{(k, n)}(q)}{\prod_{l=1}^{\min (k, n)}\left(1-q^{2 l}\right)}=\sum_{\substack{\lambda: l(\lambda) \leq \frac{1}{2} \min (k, n) \\ \sigma, \tau \in P_{R}}} c_{\sigma \mu}^{\lambda} c_{\tau \nu}^{\lambda} q^{|\lambda|}+\mathcal{O}\left(q^{\frac{1}{2} \min (k, n)+1}\right) .
$$

Proof. $\mathcal{P}(\mathfrak{p})$ is a free module over $\mathcal{P}(\mathfrak{p})^{K}$ for the pair $(G=O(n+k), K=O(n) \times$ $O(k)$ ) by Proposition 6.9. By this freeness assertion, the left side of the equation is the $q$-character of $\mathcal{P}(\mathfrak{p})$.

Proceeding exactly as in (5.6), (5.18), (5.30), and (5.42), we see that the right side of the equation agrees with the $q$-character of $\mathcal{P}(\mathfrak{p})$ up to degree $\frac{1}{2} \min (k, n)$ in $q$. That is, apply the Littlewood restriction formula for $O(n)$ and $O(k)$ (i.e., Theorem 3.5) to the decomposition in Proposition 2.3 
Proposition 6.11. Let $p_{\mu, \nu}^{(k, n)}(q)$ be the $q$-multiplicity of $V_{(k)}^{\mu} \otimes V_{(n)}^{\nu}$ in the space $\mathcal{H}_{\mathfrak{p}}$ for the symmetric pair $(S p(k+n), S p(k) \times S p(n))$. Then,

$$
\frac{p_{\mu, \nu}^{(k, n)}(q)}{\prod_{l=1}^{\min (k, n)}\left(1-q^{2 l}\right)}=\sum_{\substack{\lambda: l(\lambda) \leq \min (k, n) \\ \sigma, \tau \in P_{C}}} c_{\sigma \mu}^{\lambda} c_{\tau \nu}^{\lambda} q^{|\lambda|}+\mathcal{O}\left(q^{\min (k, n)+1}\right) .
$$

Proof. $\mathcal{P}(\mathfrak{p})$ is a free module over $\mathcal{P}(\mathfrak{p})^{K}$ for the pair $(G=S p(n+k), K=S p(n) \times$ $S p(k))$. This follows directly from the Kostant-Rallis theorem. As in the previous theorem, the left side of the equation is the $q$-character of $\mathcal{P}(\mathfrak{p})$.

The assertion follows in exactly the same way as in Proposition 6.10 with the orthogonal groups replaced by symplectic groups.

Proof of 1.22) of Theorem 1.7. The result is immediate from the fact that the right sides of the equations in Proposition 6.7 are equal, up to the appropriate order in $q$, to the right-hand sides of the equations in Propositions 6.10 and 6.11

\section{REFERENCES}

1. T. Enright and J. Willenbring, Hilbert series, Howe duality and branching rules, Preprint.

2. Roe Goodman and Nolan R. Wallach, Representations and invariants of the classical groups, Cambridge University Press, Cambridge, 1998. MR 99b:20073

3. Wim H. Hesselink, Characters of the nullcone, Math. Ann. 252 (1980), no. 3, 179-182. MR 82c: 17004

4. B. Kostant and S. Rallis, Orbits and representations associated with symmetric spaces, Amer. J. Math. 93 (1971), 753-809. MR 47:399

5. Bertram Kostant, Lie group representations on polynomial rings, Amer. J. Math. 85 (1963), 327-404. MR 28:1252

6. Dudley E. Littlewood, The Theory of Group Characters and Matrix Representations of Groups, Oxford University Press, New York, 1940. MR 2:3a

7. - On invariant theory under restricted groups, Philos. Trans. Roy. Soc. London. Ser. A. 239 (1944), 387-417. MR 7:6e

8. I. G. Macdonald, Symmetric functions and Hall polynomials, second edition, The Clarendon Press Oxford University Press, New York, 1995, With contributions by A. Zelevinsky, Oxford Science Publications. MR 96h:05207

9. N.R. Wallach and J. Willenbring, On Some q-Analogs of a Theorem of Kostant-Rallis, Canad. J. Math. 52 (2000), no. 2, 438-448. MR 2001j:22020

10. J. Willenbring, Stability properties for q-multiplicities and branching formulas for representations of the classical groups, Ph.D. thesis, University of California at San Diego, 2000.

Department of Mathematics, Yale University, 10 Hillhouse Avenue, PO Box 208283, New Haven, Connecticut 06520

E-mail address: jeb.willenbring@math.yale.edu 